\title{
Composable Finite State Machine-based Modeling for Quality-of-Information-aware Cyber-physical Systems
}

\author{
RAFAEL ROSALES and MICHAEL PAULITSCH, Intel Labs Europe
}

Time plays a major role in the specification of Cyber-physical Systems (CPS) behavior with concurrency, timeliness, asynchrony, and resource limits as their main characteristics. In addition to timeliness, the specification of CPS needs to assess and unambiguously define its behavior with respect to the other Quality-ofInformation (QoI) properties: (1) Correctness, (2) Completeness, (3) Consistency, and (4) Accuracy. Very often, CPS need to handle these QoI properties, and any combination thereof, multiple times when performing computation and communication processes. However, a model-driven and systematic approach to specify CPS behavior that jointly considers combined QoI aspects is possible but missing in existing methodologies.

As the first contribution of this work, we provide an extension to an established model of computation $(\mathrm{MoC})$ based on "Functions driven by Finite State Machine" (FunState) to enable a model-driven composition mechanism to create CPS behavior specifications from reusable components.

Second, we present a novel set of design patterns to illustrate the modeling of QoI-aware CPS specifications that can be applied in several state-of-the-art Electronic System Level (ESL) methodologies. The time semantics of the $\mathrm{MoC}$ are formalized using the tagged-signal-model, and the presented model-driven approach enables the composition of multiple design patterns.

The main benefits of the presented model-driven approach and design patterns to create CPS specifications are as follows: (a) reduce modeling effort, errors, and time through the reuse of known recipes to reincurring tasks and allow to automatically generate repetitive control flows based on extended Finite State Machines; (b) increase system robustness and facilitate the creation of holistic QoI management allowing to unambiguously define system behavior for scenarios with single/multiple QoI requirement violations in different models of computation; (c) dynamically validate timing behavior of system implementations to enable a multi-objective optimization of nonfunctional properties that influence CPS timing.

We demonstrate the aforementioned benefits through the modeling and evaluation of an infrastructureassisted automated driving case study using Infrastructure-to-Vehicle (I2V) communications to distribute QoI critical road environment information.

CCS Concepts: • Theory of computation $\rightarrow$ Automata extensions; Timed and hybrid models; $\bullet$ Computing methodologies $\rightarrow$ Modeling methodologies; $\bullet$ Computer systems organization $\rightarrow$ Embedded and cyber-physical systems; Real-time system specification; Dependable and fault-tolerant systems and networks;

Additional Key Words and Phrases: Time, timeliness, performance, quality-of-information, model of computation, moc, model-driven design, design patterns, cyber-physical systems

\footnotetext{
Authors' address: R. Rosales and M. Paulitsch, Intel Deutschland GmbH, Lilienthalstraße 15, 85579 Neubiberg; emails: \{rafael.rosales, michael.paulitsch\}@intel.com.

Permission to make digital or hard copies of part or all of this work for personal or classroom use is granted without fee provided that copies are not made or distributed for profit or commercial advantage and that copies bear this notice and the full citation on the first page. Copyrights for third-party components of this work must be honored. For all other uses, contact the owner/author(s).

(c) 2020 Copyright held by the owner/author(s).

2378-962X/2020/01-ART13

https://doi.org/10.1145/3386244
}

ACM Transactions on Cyber-Physical Systems, Vol. 5, No. 2, Article 13. Publication date: January 2021. 


\section{ACM Reference format:}

Rafael Rosales and Michael Paulitsch. 2020. Composable Finite State Machine-based Modeling for Qualityof-Information-aware Cyber-physical Systems. ACM Trans. Cyber-Phys. Syst. 5, 2, Article 13 (January 2021), 27 pages.

https://doi.org/10.1145/3386244

\section{INTRODUCTION}

Cyber-physical Systems (CPS) can be described as systems with integrated physical and computational processes. CPS provide the capability to jointly coordinate the sensing, processing, communication, storage and actuation of physical and computational processes that enables the monitoring and control of both the physical and information world including human interaction in the loop. This implies that strict functional and nonfunctional requirements must be met by CPS to perform the aforementioned tasks successfully.

Timeliness is one key requirement of CPS, as CPS are often reactive systems, which interact with the environment at a speed imposed by the environment, and have the following real-time characteristics [43]: (a) concurrency of multiple time-overlapping communication/computations processes; (b) time constraints on the latency of communication/computation processes defined by the application requirements; (c) asynchrony where processes have the inability to predict environment behavior; and (d) implementation resource limits such as time, energy, memory, and so on.

Following state-of-the-art methodologies for the design of CPS [44], a system specification compliant with the system requirements is first created and verified before the creation of the CPS implementation that is then validated against its specification. This design flow may be iterated for (a) describing more granular specifications of hierarchical CPS and/or (b) to feedback necessary changes to the specification when necessary. The separation of system specification from implementation allows us to explore different alternative realizations of the desired functionality with possibly different tradeoffs in nonfunctional properties. This means that the specification of CPS should meet timeliness requirements independently of the CPS implementation. However, as no physical system can be guaranteed to be perfectly accurate, secure, and free from failures, the specification of CPS needs to unambiguously define the behavior of CPS processes in response to deviations of the system implementation from its specification or on the environment assumptions.

This applies to the timeliness aspect, where a system' failure to synchronize or to comply with hard-real time requirements may require to immediately transition the system into a safe state. Equally important, it is necessary to specify the CPS processes' behavior with respect to the compliance/deviation on the other four Quality-of-Information (QoI) properties [48]: (1) information correctness, where monitoring and validation of data is performed for error detection to, for example, apply error correction; (2) information completeness, for data validation such as the identification of "known unknowns" in the perception of the system against a model of reality; (3) information consistency, where analysis on the correlation of data can be performed to, e.g., evaluate different sources of information to improve CPS reliability and safety; and, finally, (4) information accuracy, where the deviation of data from the ground truth is evaluated and used to, e.g., perform correcting actuator actions.

All relevant QoI properties of a CPS process should be considered in conjunction with the behavior specification as CPS implementations may achieve different tradeoffs in the data QoI properties. For example, error detection/correction, may require to consume some amount of the time budget available or less accurate data could be produced in a smaller amount of time. 
The specification of QoI-aware CPS behavior is also important if systems are implemented using components (HW or SW) that even under ideal circumstances cannot provide any QoI guarantees. The new standard ISO/PAS 21448 known as SOTIF [28] aims to provide guidance in the creation of systems with no unreasonable risk due to functional specification insufficiencies or reasonable misuse even in the absence of malfunctions and QoI-aware behavior specifications can be part of the means to achieve this goal.

Problem statement: In this article, we address two challenges in the specification of CPS:

The first challenge is to be able to describe complex system behaviors and to keep the model components consistent ${ }^{1}$ [14], as different systems may reuse previously designed components plus some modifications and thus creating a divergence of several component versions.

At the same time, and present in all modeling paradigms, the modeling of computation and communication processes requires to frequently solve a set of general problems manifested at multiple phases in a process and at multiple levels of abstraction. Such generic problems have been typically addressed in so-called design patterns, which can be applied to specific problems with the appropriate customization to the problem context but providing the designer with a proven recipe to address his problem at hand more efficiently.

Each QoI property relevant to a CPS process represents such a general problem to be addressed multiple times and in different combinations that, to the best of our knowledge, are missing in the set of known design patterns. The description of reusable design patterns of runtime monitors for the creation of QoI-aware behavior specifications is the second challenge addressed in this article. ${ }^{2}$

The two main contributions of this work to address the aforementioned challenges are as follows:

- A model-driven formalism to enable the specification of composable descriptions of CPS processes behavior that enables the reuse of actor-oriented components.

- A novel set of five composable design patterns for the modeling of QoI-aware CPS specifications that can be applied in several state-of-the-art Electronic System Level (ESL) methodologies.

The target of the model-driven approach is a process specification using a generalization of the "Functions driven by Finite State Machines" (FunState) model of computation (MoC) that allows functions to share variables and embedded with time semantics. The proposed model-driven formalism provides an extension to the MoC to enable a syntactic verification of actor composition, including the control flow. In the work at hand, semantic or behavioral verification is not demonstrated; however, the introduced modeling elements establish the base for future work in this respect. The target $\mathrm{MoC}$ can be used or adapted in multiple system level methodologies by defining appropriate transformation rules to their semantics, for example, AADL [41], PtolemyII [16], or for Intel CoFluent [10] as in Reference [40].

The benefits of the presented model-driven approach to create CPS specifications are as follows:

- Reduce modeling effort, errors and time through the reuse of behavior specifications and allow to automatically generate patterns of control flows specified by extended Finite State Machines (FSMs).

- The chosen formalism allows complex CPS specifications in several MoCs: Hierarchical Concurrent FSMs [12], dataflow, continous-time, time-triggered, and discrete event.

\footnotetext{
${ }^{1}$ The term consistency is used here in the context of Reference [14] and is not related to the QoI property.

${ }^{2}$ Note that the proposed design patterns perform runtime checks on the quality of information and not on design errors.
} 
- Existing ESL methodologies can be used to evaluate CPS implementations to validate the fulfillment of the system specification for both functional and nonfunctional properties.

The benefits of the presented design patterns for QoI-aware CPS specifications are as follows:

- avoid incomplete CPS specifications and facilitate the creation of holistic QoI management allowing to unambiguously define system behavior for scenarios with single/multiple QoI requirements violations,

- dynamically validate timing behavior of system implementations to enable a multi-objective optimization of nonfunctional properties that influence CPS timing.

To the best of our knowledge, there is no existing work addressing the specification of QoI-aware behavior of CPS processes based on reusable and composable design patterns.

The rest of the article is organized as follows: Sections 2 and 3 provide the formal definitions of the modeling elements and time semantics of the existing target $\mathrm{MoC}$ and novel source MoC respectively. Section 4 introduces the rules for the model-driven specification of composable CPS behavior for the source MoC. Section 5 provides state-of-the-art languages, approaches and design patterns for modeling real-time CPS. In Section 6, a set of novel design patterns for each QoI is provided. Section 7 illustrates the composition of timeliness with the other design patterns. Section 8 presents a I2V CPS case study where the presented approach has been applied. To finalize, Section 9 summarizes the main contributions.

\section{TIMED ACTOR-ORIENTED MODELING FORMALISM}

In the proposed model-driven generation of CPS behavior specifications, two formalisms are used: The target formalism describes the output of the model-driven approach, which is the actororiented model whose elements are described in this section. The source formalism, provided in the next section, enables to specify composable actor-oriented models where the control flow of actors can be created by composing the FSMs of simpler specifications.

\subsection{Target Formalism: TimedSvFunState}

The target modeling formalism is based on the existing actor-oriented language SysteMoC [17]. SysteMoC extended the FunState MoC [47] to include shared variables. The time semantics for a SysteMoC model proposed in MAEstro [38] are adopted as well. In the rest of the article, the SysteMoC model of computation extended with time semantics will be referred to as TimedSvFunState.

In the following, the definition of the actor-oriented elements and time semantics are summarized.

Definition 2.1 (Actor). An actor $r$ is a tuple $r=\left(\mathcal{I}^{*}, O^{*}, \mathcal{A}, \mathcal{M}, \mathcal{V}\right)$ containing a set of input ports $\mathcal{I}^{*}$, a set of output ports $O^{*}$; a set of methods $\mathcal{A}$ comprised of actions and functional guards; a Finite State Machine $\mathcal{M}$ orchestrating the execution of actions and the reading/writing to ports; a set of local variables $\mathcal{V}$ accessible by all actor methods, where each variable $v \in \mathcal{V}$ has an initial value denoted as $v^{i}$ and a current value $v^{c}$.

Actors model functional entities and can operate concurrently or be scheduled by some external entity and may only communicate by exchanging tokens through message passing via their input and output ports. See Figure 1 for an example and graphical representation of the actor elements.

Definition 2.2 (Token). A token $e$ is either an instance of a data structure $y$ or a pointer/reference to an object $y$ with unique ownership to it, i.e., tokens cannot be shared by multiple owners. 


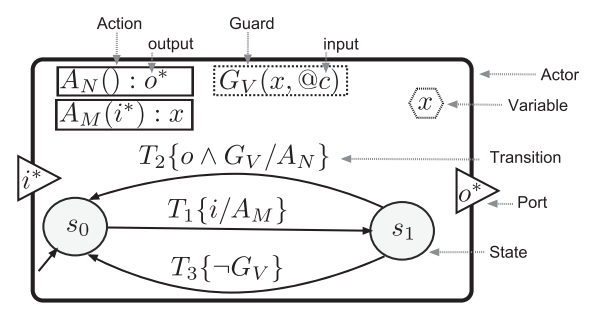

The actor shown at the left represents a processing task, e.g., a Fast Fourier Transform, in which data is read from input port $i^{*}$ and processed by action $A_{M}$, e.g. transforming the data to the frequency domain. An output token with the result is generated through action $A_{N}$ only if a) the guard $G_{V}$ returns true, e.g., by filtering $x$ values smaller than a threshold $c$, and $\mathrm{b}$ ) if there exist an available slot at the FIFO connected to output port $o^{*}$

Fig. 1. Actor elements and their graphical representation. A FSM drawn at the bottom synchronizes I/O and the execution of actions based on activation patterns (a predicate of guards) defined at each FSM transition.

Input ports and output ports receive and produce tokens, can be interconnected through a single message queue with First-In-First-Out (FIFO) semantics, i.e., no tokens can be lost, are delivered in-order and if the queue is full, it will block further execution of the actor sending the token.

Definition 2.3 (Message Queue). A message queue $q$ is a FIFO buffer defining an unidirectional channel with both an actor output port $o^{*} \in r_{i} . O^{* 3}$ and an actor input port $i^{*} \in r_{j} . \mathcal{I}^{*}$ connected to it and denoted as $o^{*} \mapsto q$ and $q \mapsto i^{*}$ respectively. A message queue contains an associated channel parameter function $\mathcal{P}: q \rightarrow \mathbb{N}_{\infty} \times E^{*}$ that associates with each channel $q$ its buffer size $n \in \mathbb{N}_{\infty}=1,2,3, \ldots \infty$, and possibly also a nonempty sequence $E \in E^{*}$ of initial tokens $e$ of type $y$, where $E^{*}$ denotes the set of all tuples of finite sequences.

The execution of actions, writing and reading of tokens by an actor is specified by its FSM:

Definition 2.4 (FSM). The FSM $\mathcal{M}$ of an actor $r$ is a tuple $\mathcal{M}=\left(S, s_{0}, \bar{s}, T\right)$ containing a finite set of states $S$, an initial state $s_{0} \in S$, an active state $\bar{s} \in S$, and a finite set of state transitions $T \subseteq S \times S$.

Definition 2.5 (Transition). A transition $t \in T$ is a tuple $t=\left(s^{s}, s^{d}, k, A\right)$. If the transition source state $s$ is the current active state $\bar{s}$ and the activation pattern $k$ evaluates to true, then the transition and the containing actor are said to be enabled, ${ }^{4}$ and if fired, the transition is taken by performing (a) the ordered execution of the set of actions $A \subset \mathcal{A}$, (b) the consumption of tokens at the input ports specified by the input guards in the activation pattern $k$ and the production of tokens at the output ports as specified by the output guards in the activation pattern $k$ (in any order), and (c) the FSM active state is set to the destination state $s^{d}$. The transition is considered complete when all these steps are performed and only then could another transition be fired in this FSM.

Definition 2.6 (Activation Pattern). A transition activation pattern $k$, or firing rule, consists of the logical conjunction of a set of input, output, and functionality guards composing a Boolean function $k: \mathbb{N}^{\left|I^{*}\right|} \times \mathbb{N}^{\left|O^{*}\right|} \times E^{\left|I^{*}\right|} \times \mathcal{V} \times C \rightarrow\{$ true, false $\}$, where $|\mathrm{X}|$ denotes the set cardinality of input or output ports and $E$ is a token sequence at the input ports, conditioning the enabling of an FSM transition to the number and values of tokens at its input queues, the number of free slots at its output queues, the actor internal state $\mathcal{V}$, and to a set of transition specific constant parameters $C .^{5}$

The following three definitions provide the basic elements to specify an activation pattern:

Definition 2.7 (Input Guard). An input guard $\# i_{n}(m)$ is a function $i_{n}: \mathbb{N} \rightarrow\{$ true, false $\}$, which encapsulates a test condition on the availability of tokens $m \in \mathbb{N}$ at input port $i_{n}^{*} \in \mathcal{I}^{*}$.

\footnotetext{
${ }^{3}$ The dot notation is used to indicate a member of an object.

${ }^{4}$ An enabled actor can be fired immediately or when specified by a scheduler.

${ }^{5}$ Constant parameters are immutable variables used to define parameterized transitions. This allows us to specify transitions dynamically, for example, inside a for loop where the iteration index is used at each transition as a constant parameter.
} 
Definition 2.8 (Output Guard). An output guard $\# o_{n}(m)$ is a function $o_{n}: \mathbb{N} \rightarrow\{$ true, false $\}$ encapsulating a test condition on the number of available slots $m \in \mathbb{N}$ at output port $o_{n}^{*} \in O^{*}$.

To simplify notation, an input/output guard $\# i_{n}(1) / \# o_{m}(1)$ is simply depicted as $n$ or $m$, i.e., with the id of the respective input/output port $i_{n}^{*} / o_{n}^{*}$. If no parameter is given, then it is assumed to equal 1.

Definition 2.9 (Functionality Guard). A functionality guard $g$ is a function $g: \mathcal{V} \times C \times E^{\left|I^{*}\right|} \rightarrow$ \{true, false\}. It encapsulates a test condition on the tokens sequences $E^{\left|I^{*}\right|}$ on input ports $\mathcal{I}^{*}$, the local variables $\mathcal{V}$, and constant parameters $C{ }^{5}$ A functionality guard is side-effect free.

In Figure 1, a transition labeled $T_{1}$ has an activation pattern comprised of a single input guard $i$ together with the functionality guard $G_{T}$. Neither transitions $T_{1}$ or $T_{3}$ have an ouput guard, which indicates that there is no requirement on available output slots for the transition to take place. $T_{2}$ in contrast includes an action to write an output token, and thus an output guard is necessary.

In contrast to functionality guards, actions of an actor may modify the current value of local variables and/or produce tokens at the actor outputs:

Definition 2.10 (Action). An action $a \in A$ is a method or function $a: \mathcal{V} \times C \times E^{\left|I^{*}\right|} \rightarrow \mathcal{V} \times E^{\left|O^{*}\right|}$, where $E$ is a token sequence at the input or output ports, $|X|$ denotes the set cardinality of input or output ports, $\mathcal{V}$ is the set of local variables and $C$ is a set of constant parameters ${ }^{5}$.

Actions and functionality guards can be understood as methods of a programming language, and they can be used as placeholders to specify the method interface alone, i.e., only define the inputs and outputs, or also include the implementation as well.

To enable the hierarchical composition of actors, and thus enhance significantly the scalability of specifications, e.g., to implement HCFSMs [12], a cluster is defined:

Definition 2.11 (Cluster). A cluster is a directed bipartite graph $c=\left(\mathcal{I}^{*}, O^{*}, X, D\right)$ containing a set of cluster input ports $\mathcal{I}^{*}$ and cluster output ports $O^{*}$, a set of vertices $X=Q \cup R \cup C$ partitioned into message queues $Q$, actors $R$ and subclusters $C$, a set of directed edges $d=\left(x_{s r c}, x_{s n k}\right) \in$ $D \subseteq\left(\left(Q \cup \mathcal{I}^{*}\right) \times\left(R . \mathcal{I}^{*} \cup C . \mathcal{I}^{*}\right)\right) \cup\left(\left(R . O^{*} \cup C . O^{*}\right) \times\left(Q \cup O^{*}\right)\right)$ from queues $q \in Q$ or cluster input ports $i^{* *} \in \mathcal{I}^{*}$ to actor or subcluster input ports $i^{*} \in R . I^{*} \cup C . I^{*}$ as well as from actor or subcluster output ports $o^{*} \in\left(R . O^{*} \cup C . O^{*}\right)$ to queues or cluster output ports $o^{* *} \in O^{*}$.

A cluster enables to encapsulate a set of interconnected actors into a single entity, which only exposes relevant input and output ports connected to the inner elements.

\subsection{Time Semantics}

In the specification of CPS, three time models can be identified, as proposed in Reference [19], according to how the physical time (continous time dimension in which physical process evolve, a.k.a. elapsed real time, or wall-clock time) and the logical time (discrete time dimension in which information processes evolve) relationship is defined:

- Asynchronous model: Where the implementation's execution platform, including the scheduling policy, HW performance, and the utilization ratio of processors, determines the evolution of logical time. Specifications in this model are platform dependent, with temporal non-determinism and recur to the specification of deadlines used to fix execution bounds. This model is the most commonly used in Java, ADA, and C real-time libraries.

- Preestimated time model: Execution time information is provided a priori, by annotating tasks with timing estimations to allow analysis about timing behavior. In this model, the specification can be evaluated on multiple implementations through the use of different 
annotation values. This is often used in formal languages such as Timed Automata [1] or Giotto [25].

- Synchronous time model: This model assumes that the implementation execution platform is fast enough to complete the execution of tasks before the arrival of the next environment event. Esterel [7], Lustre [23], and Signal [21] are languages using this model.

To express time in the actor model the tag signal model [33] is used: An order is induced on events by associating them with a tag $\mathcal{T} \in \mathbb{T}$, where $\mathbb{T}$ is a tag set to be defined. An event $z$ is defined as a tuple $(u, \mathcal{T})$ of an event id $u$ and a tag $\mathcal{T}$, i.e., $z \in U \times \mathbb{T}$, where $U$ is the set of event ids.

In the target formalism, three classes of events are defined: (1) creation and consumption of tokens, (2) start and end of execution of an actor action, and (3) start and end of an FSM transition.

The tag system used in this work corresponds to the semantics of superdense time [33] (also used in discrete-event simulation engines) and is presented next:

Definition 2.12 (Timed Actor Model Tag System). $\mathbb{T}$ is a totally ordered set $\mathbb{T}=\mathbb{R}_{0}^{+} \times \mathbb{N}$, where each event has a $\operatorname{tag} \mathcal{T}(\tau, j) \in \mathbb{T}$, denoting that the event took place at a particular point in time (physical time) with a time stamp $\tau \in \mathbb{R}_{0}^{+}$and has a unique index $j \in \mathbb{N}$ (logical time). The resulting order relation for $\mathcal{T}_{1}, \mathcal{T}_{2} \in \mathbb{T}$ with $\mathcal{T}_{1}=\left(\tau_{1}, j_{1}\right)$ and $\mathcal{T}_{2}=\left(\tau_{2}, j_{2}\right)$ is then given as $\mathcal{T}_{1}<\mathcal{T}_{2} \Longleftrightarrow \tau_{1}<$ $\tau_{2} \vee\left(\tau_{1}=\tau_{2}\right) \wedge\left(j_{1}<j_{2}\right)$.

The unique index $j$ of an event is monotonically increased in the execution of a model process, and represents the real order in which events where processed. The time stamp tag (or simulated time stamp) is assigned to each event as follows:

FSM transition start/end events. The start time of a transition depends on three conditions: (a) the activation pattern, (b) the scheduling of the transition's actor, and (c) the scheduling of the transition. The start time stamp tag of a transition is equal to the point in time of its scheduling. The end time stamp tag of a transition is equal to the start time tag plus the duration of all referenced actions.

Action start/end events. The start time of an action depends on (a) the scheduling of the action's transition and (b) the order in which the action is referenced in the transition. The start time stamp tag is equal to the point in time of scheduling of the transition plus the duration of all previous actions in the transition. The time stamp tag of the end of an action is equal to the action start tag plus the time duration of the action.

Time duration of actions. Each individual execution $\hat{a}$ of an action can be annotated with a time duration $\tau^{d}$ indicating the difference in the time stamp of the initiation of execution $\tau^{i}$ and finalization of execution $\tau^{f}$. This annotation can be static or dynamic, but must always be set before the scheduling of the transition to consider a new value.

Token creation and consumption events. Tokens are generated or consumed in the same time stamp tag as the action's end time stamp generating or consuming them respectively. Equivalently, from the message queue perspective, tokens in a message queue are always tagged with time stamps of arrival time $\tau^{a}$ and dispatching time $\tau^{p}$. There is no time duration explicitly associated in the FIFO queue as the defined semantics describe instantaneous communication between actors. ${ }^{6}$

The TimedSvFunState MoC is capable to express the three time models and even continuous time process [38] by selecting an appropriate scheduling policy and an appropriate annotation of action time duration: For example, static time annotations enable to model the preestimated

\footnotetext{
${ }^{6}$ Non-instantaneous communication can be modeled through extra actors representing communication processes.
} 
time model. If the scheduling policy considers contention and arbitration overheads of the system implementation, then the asynchronous time model is used. Finally, if the scheduling policy can guarantee the completion of the actions before new events are triggered, then the synchronous time model can be utilized. This MoC also allows us to model local clocks to specify subcomponent synchronization mechanisms.

\section{SOURCE FORMALISM: NOVEL ELEMENTS}

\subsection{New Elements for Transition Refinement and FSM Composition}

In this section, a set of novel modeling elements are presented to extend the TimedSvFunState MoC that enable a model-driven creation of composable actor-oriented behavior specifications. This MoC will be referred to as LabeledFunState.

First, we introduce the notion of an id $\mathcal{N}$ for every element defined here and in Section 2 to semantically match elements of two components. ${ }^{7}$ Second, a partial FSM is defined to enable the specification of an actor FSM from a composition of simpler, and possibly incomplete, parts:

Definition 3.1 (Partial FSM). A partial FSM $\mathcal{M}^{p}$ is a tuple $\mathcal{M}^{p}=(S, T)$ containing a finite set of states $S$ and a finite set of state transitions $T$.

Note that, compared to Definition 2.4 of an FSM, a partial FSM does not define an initial state, nor does it define an active state, as it is not assumed that a partial FSM can be used by an actor before composing it with another partial FSM.

A partial FSM references input/output ports, local variables, actions, and guards. Thus, a partial actor is defined to bundle a partial FSM together with the aforementioned referenced elements:

Definition 3.2 (Partial Actor). A partial actor $r^{p}$ is a tuple $r^{p}=\left(\mathcal{I}^{*}, O^{*}, \mathcal{A}, \mathcal{M}^{p}, \mathcal{V}\right)$ containing a set of input and output ports: $\mathcal{I}^{*}, O^{*}$; a set of methods $\mathcal{A}$ comprised of actions and functional guards; a partial Finite State Machine $\mathcal{M}^{p}$ orchestrating the execution of actions and the reading/writing to ports; and a set of local variables $\mathcal{V}$ that are shared by all the partial actor's methods.

Third, to enable the possibility to recursively refine behavior through composition, we propose an extended definition of a FSM transition from the fixed structure: Source state $\rightarrow$ activation pattern $\rightarrow$ ordered list of actions $\rightarrow$ Destination state to the less constrained: Source state $\rightarrow$ Any converging combination of transition paths $\rightarrow$ Destination state. For this, two types of transitions are defined:

Definition 3.3 (Atomic Transition). An atomic transition is a transition as stated in Definition 2.5.

Definition 3.4 (Non-Atomic Transition). A non-atomic transition $t_{N A}$ is a tuple $\left(s^{s}, s^{d}, \mathcal{M}^{n}\right)$. It defines a source $s^{s}$, a destination state $s^{d}$, and a placeholder $\mathcal{M}^{n} . \mathcal{M}^{n}$ stands for either (a) an atomic transition, (b) a chain of atomic and/or non-atomic transitions, or (c) as an empty placeholder.

A non-atomic transition allows us to replace a single atomic transition with a more granular model consisting of several transitions. Partial actors that have a single source and destination states, are useful for refining non-atomic transitions and are called Single-Entry-Single-Exit (SESE) partial actors and define two special states that can be labeled as source state $s^{s} \in S$ and destination state $s^{d} \in S$, and constraint all finite transition paths to start at $s^{s}$ and end at $s^{d}$, i.e., loops are allowed. Figure 2 shows an example where a non-atomic transition (left) can be refined with a SESE (right).

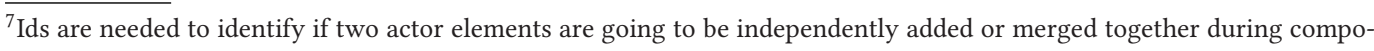
sition.
} 


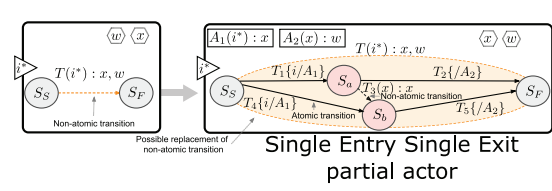

In this example, a non-atomic transition is replaced with a set of converging transition paths. The original transition only specifies that the variables $x$ and $w$ can be modified. The refined non-atomic transition specifies exactly what actions will be performed. In this case, two guards split the control flow, allowing for three different possible computations: $\left(T_{1} \succ T_{2}\right)$ or $\left(T_{1} \succ T_{3} \succ T_{5}\right)$ or $\left(T_{4} \succ T_{5}\right)$

Fig. 2. A Non-atomic transition is a placeholder for either a single or a chain of atomic transition.

To create an actor from a partial actor, it is necessary and sufficient to add the following elements:

Definition 3.5 (Partial Actor to Actor Conversion). A partial actor can be transformed into an actor if (1) an initial state is specified and (2) all non-atomic transitions placeholders are provided with either an atomic transition or a chain of atomic and/or non-atomic transitions.

With the previously introduced definitions, it is possible to split an actor model specification in smaller sub-components. However, to enable the validation of a composition of these subcomponents, it is necessary to enhance the TimedSvFunState meta-model to include the domains of variables and tokens, as well as the side-effects of actions and transitions on those. These extensions to the TimedSvFunState specification are provided in the next subsection.

\subsection{Data Flow Transformation Specification}

To enable actor composition and address the reusability problem of control flow logic expressed as FSMs, the following extensions are proposed to specifications in the LabeledFunState MoC:

- include the explicit specification of valid variable domains, including the domains of the received and transmitted tokens. A domain may include the set " $\emptyset$ " for non-assigned variables. ${ }^{8}$

- include the explicit specification of allowed dataflow transformations on each FSM action/ transition as well as required invariants.

- include the explicit specification of expected arrival of tokens from the environment after executing action/transitions and/or at states with no incoming transitions.

The aforementioned extensions are formalized in a so-called dataflow transformation specification:

Definition 3.6 (Dataflow Transformation Spec.). A dataflow transformation specification of a partial actor $r^{p}$ is a tuple $(v, \rho, \alpha, \eta, \psi, \Psi)$, where $v$ is a binary relation between the local variables $r^{p} . \mathcal{V}$ and the set of possible domains ${ }^{9} V \mid \nu \cdot v^{c} \in V ; \rho$ is a binary relation between the ports $r^{p} . P$ and the set of possible domains of their tokens $T ; \alpha$ is a binary relation between the variables $r^{P}$. $\mathcal{V}$ and their initial value $v \cdot v^{i} \in D ; \eta$ is a binary relation between the input ports $r^{p} . \mathcal{I}^{*}$ and a sequence of expected tokens $E$ at states with no incoming transitions; $\psi$ is a binary relation between the actions $r^{P}$. $\mathcal{A}$ and a specification $\phi$ of allowed side-effects on variables, input and output port tokens and expected input tokens; $\Psi$ is a binary relation between the transitions $r^{p} . T$ and a specification $\phi$ of allowed side-effects on variables, input and output port tokens, and expected input tokens.

Figure 3 shows a partial actor example with its corresponding dataflow transformation spec.

Definition 3.7 (Specification of Allowed Side-Effect). An action or transition specification $\phi$ of allowed side-effects is a set of binary relations from the domain and the range of each (1) variable,

\footnotetext{
${ }^{8}$ Notation used for a variable $d$, which could be assigned to an element of $D$ or not assigned at all: $d \in D \cup \emptyset$.

${ }^{9}$ The domain of variables or ports may be left abstractly specified, i.e., the concrete elements of the set may not be fixed to enable the specification of type-agnostic processes, which may be refined during composition with other partial actors.
} 


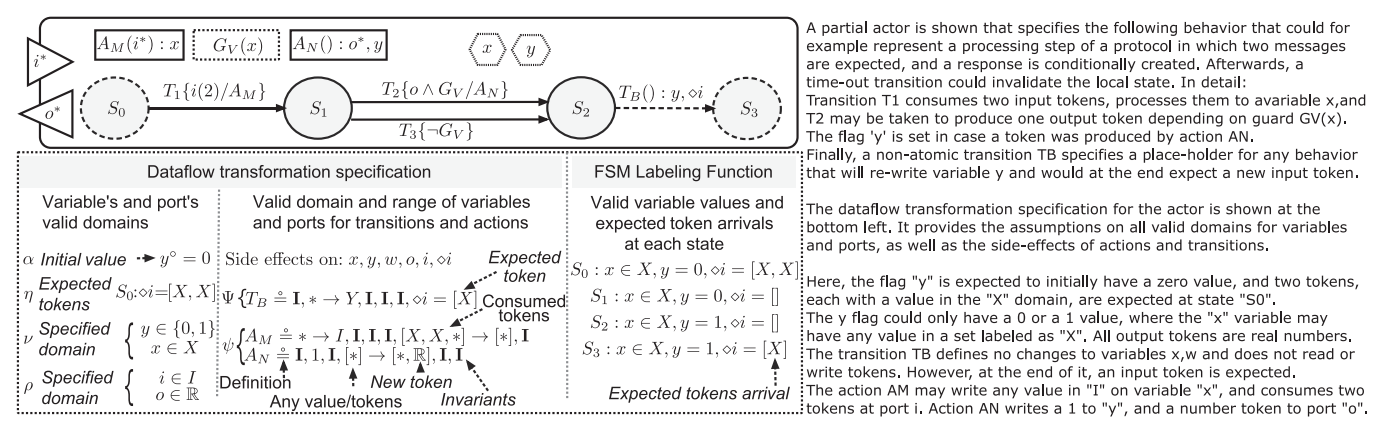

Fig. 3. Top left: An example of a partial actor. Bottom left: Specification of its dataflow transformation consisting of (a) initial values and overall domains and (b) valid domain and range of variables/tokens for actions and transitions. The valid domains at each state can be computed from the dataflow transformation spec.

(2) input and output port tokens, and (3) expected input tokens of a partial actor $r^{p}$. This includes the special case of the identity binary relation that assigns to each element of the domain the exact same element in the range to specify invariants, i.e., variables/ports that are left unaltered.

The side-effects of actions and transitions need to indicate not only the domain and range of modified variables, but also to indicate the ones that remain invariant after their execution. This ensures that no unexpected side-effects could occur during the composition of several partial actors.

The explicit inclusion of expected tokens from the environment addresses the side-effects performed by a partial actor on the environment, i.e., on the connected actors. It is not the intent to define a priori all possible tokens that may arrive from an unknown environment, but rather to encode the effects that the environment may produce as the consequence of the local action that caused them. For example, if a timer actor is connected to delegate the monitoring of a time-out alert, then the action of writing a request token to this timer actor will have the effect of a response token after the time-out has ellapsed, and it is meaningful to encode this information in the subsequent FSM states after the request, so that the response can be handled appropriately.

From the dataflow transformation specification, it is possible to infer the semantic meaning of FSM states and generate a set of propositions $A P$ that reflect the "current situation" being represented by an FSM state in the state-space of the partial actor, i.e., the possible values of local variables, port tokens, and known environment state. These propositions are used to assign a label $\lambda$ to each state, through an FSM labeling function $\mathcal{L}: S \rightarrow 2^{A P}$, to transform the FSM into a so-called Kripke structure, i.e., a labelled state-transition graph [8]. Such FSM labeling functions have been used in the context of model-checking, for example in the temporal logic of actions [31]. At the bottom of Figure 3 the resulting FSM labeling function is shown, where each state defines the possible values of the partial actor variables and expected tokens.

\section{COMPOSITION OF BEHAVIOR SPECIFICATIONS}

In this section, a set of rules are given to enable a valid transformation and composition of actororiented behavior specifications using the elements defined in Section 3.

\subsection{Partial Actor Composition Rules}

Partial actors can only be composed together by following a set of rules to ensure that the composed FSMs states and transition are compatible. Here, compatible means that the valid domain and ranges of the variables and ports remain consistent in the merged FSM. 
In general, to compose any two partial actors at a time, a set of transformation operations is required to (a) prepare each partial actor before the composition and (b) compose the two partial actors. The former is a set of unitary operations that are necessary, because it is not always possible to just "add" or "merge" together actor elements, due to id collisions or discrepancies, respectively. The latter is a set of binary operations that together define the composition of partial actors.

We categorize a set of allowed transformation operations to adapt and to compose the structure of the two partial actor sources:

- [Unitary operations] Change the id of existing elements of an existing partial actor (states, variables, ports, transitions, methods).

- [Binary operations] Direct aggregation of disjoint elements (states, variables, ports, transitions, methods) that do not require any modification during composition.

- [Binary operations] Mapping of elements to be merged (states, variables, ports, transitions, methods) to combine two elements into a single one.

The rules for each operation category is given the following:

4.1.1 Renaming Rules. The id of any element of a partial actor can be renamed, as long as the new id does not collide with an existing id of an element of the same type, and all references to the renamed elements are updated as well:

Renaming Rule 1 (Renaming States). The id $\mathcal{N}$ of a state $r^{p}$.s can be renamed to a new id $\mathcal{N}_{2}$, if: (1) the new id is not already an id of an existing state in the partial actor FSM, i.e., $\mathcal{N}_{2} \notin \bigcup_{i}\left(r^{p} . s_{i} . \mathcal{N} \mid r^{p} . s_{i} \in r^{p} . S\right) ;(2)$ the source states of all FSM transitions referencing this state are updated with the new id, i.e., $r^{p} . s . \mathcal{N}=\mathcal{N}_{2} \Rightarrow\left(\forall s^{s} \in \bigcup_{j}\left(t_{j} . s^{s} \mid t_{j} . s^{s} . \mathcal{N}==r^{p} . s . \mathcal{N}\right): s^{s} . \mathcal{N}=\mathcal{N}_{2}\right)(3)$ the destination states of all FSM transitions referencing this state are updated with the new id, i.e., $r^{p} . s . \mathcal{N}=\mathcal{N}_{2} \Rightarrow\left(\forall s^{d} \in \bigcup_{j}\left(t_{j} . s^{d} \mid t_{j} . s^{d} . \mathcal{N}==r^{p} . s . \mathcal{N}\right): s^{d} . \mathcal{N}=\mathcal{N}_{2}\right)$.

Renaming Rule 2 (RenAming VARiables). The id $\mathcal{N}$ of a variable $r^{p} . v$ can be renamed to a new id $\mathcal{N}_{2}$, if: (1) the new id is not already an id of an existing variable in the partial actor, i.e., $\mathcal{N}_{2} \notin \bigcup_{i}\left(r^{p} . v_{i} . \mathcal{N} \mid r^{p} . v_{i} \in r^{p} . \mathcal{V}\right) ;(2)$ all the methods referencing this variable are updated with the new id; i.e., $r^{p} . v \cdot \mathcal{N}=\mathcal{N}_{2} \Rightarrow\left(\forall v \in \bigcup_{j}\left(m_{j} \cdot v \mid m_{j} \cdot v \cdot \mathcal{N}==r^{p} \cdot v \cdot \mathcal{N}\right): v \cdot \mathcal{N}=\mathcal{N}_{2}\right)$; (3) the new id is updated in the dataflow transformation specification $(v, \alpha, \psi, \Psi)$ for the variable domain, variable initial value, and methods and transitions side-effects.

Renaming Rule 3 (Renaming Ports). The id $\mathcal{N}$ of a port $r^{p} . p \in\left\{r^{p} . \mathcal{I}^{*} \cup r^{p} . O^{*}\right\}$ can be renamed to a new id $\mathcal{N}_{2}$, if: (1) the new id is not already an id of an existing port in the partial actor, i.e., $\mathcal{N}_{2} \notin$ $\cup_{i}\left(r^{p} \cdot p_{i} \cdot \mathcal{N} \mid r^{p} \cdot p_{i} \in\left\{r^{p} \cdot \mathcal{I}^{*} \cup r^{p} . O^{*}\right\}\right) ;(2)$ all input and output guards are updated with the new id; (3) all the methods referencing this port are updated with the new id; i.e., $r^{p} . p . \mathcal{N}=\mathcal{N}_{2} \Rightarrow(\forall p \in$ $\left.\bigcup_{j}\left(m_{j} \cdot p \mid m_{j} \cdot p \cdot \mathcal{N}==r^{p} . p \cdot \mathcal{N}\right): p . \mathcal{N}=\mathcal{N}_{2}\right) ;(4)$ the new id is updated in the dataflow transformation specification $(\rho, \eta, \psi, \Psi)$ for the port domain, initial input port expected tokens, and methods and transitions side-effects.

Renaming Rule 4 (Renaming Methods). The id $\mathcal{N}$ of a method $r^{p} . m$ can be renamed to a new id $\mathcal{N}_{2}$, if the new id is not already an id of an existing method in the partial actor, i.e., $\mathcal{N}_{2} \notin \bigcup_{i}\left(r^{p} . m_{i} \cdot \mathcal{N} \mid r^{p} . m_{i} \in r^{p} . \mathcal{M}\right)$.

Renaming Rule 5 (Renaming Transitions). The id $\mathcal{N}$ of a transition $r^{p}$. M.t can be renamed to a new id $\mathcal{N}_{2}$, if the new id is not already an id of an existing transition in the partial actor FSM, i.e., $\mathcal{N}_{2} \notin \bigcup_{i}\left(r^{p}\right.$.M. $t_{i} . \mathcal{N} \mid r^{p}$.M. $\left.t_{i} \in r^{p} . T\right)$.

4.1.2 Direct Aggregation Rules. The following rules indicate the condition for states, variables and ports for being aggregated when modification is not necessary in a partial actor composition. 
Aggregation Rule 1 (Copy Unioue Elements). Each element to be aggregated from two actor sources $r_{1}$ and $r_{2}$, i.e., each state $s$, each variable $v$, each port $p$, each method $m$, and each transition $t$, must have a unique id $\mathcal{N}$ not existing on the other actor to become an element of the resulting actor $r_{c}$,

$$
\begin{aligned}
& \forall s_{i} \forall s_{j}:\left(s_{i} \in r_{1} . S_{u} \wedge s_{j} \in r_{2} . S_{u} \wedge s_{i} . \mathcal{N} \neq s_{j} . \mathcal{N} \Rightarrow s_{i}, s_{j} \in r_{c} . S\right) \\
& \forall v_{i} \forall v_{j}:\left(v_{i} \in r_{1} \cdot \mathcal{V}_{u} \wedge v_{j} \in r_{2} \cdot \mathcal{V}_{u} \wedge v_{i} . \mathcal{N} \neq v_{j} . \mathcal{N} \Rightarrow v_{i}, v_{j} \in r_{c} . \mathcal{V}\right) \\
& \forall p_{i} \forall p_{j}:\left(p_{i} \in r_{1} . P_{u} \wedge p_{j} \in r_{2} . P_{u} \wedge p_{i} . \mathcal{N} \neq p_{j} . \mathcal{N} \Rightarrow p_{i}, p_{j} \in r_{c} . P\right) \\
& \forall m_{i} \forall m_{j}:\left(m_{i} \in r_{1} . \mathcal{M} \wedge m_{j} \in r_{2} . \mathcal{M} \wedge m_{i} . \mathcal{N} \neq m_{j} . \mathcal{N} \Rightarrow m_{i}, m_{j} \in r_{c} . \mathcal{M}\right) \\
& \forall t_{i} \forall t_{j}:\left(t_{i} \in r_{1} . T \wedge t_{j} \in r_{2} . T \wedge t_{i} . \mathcal{N} \neq t_{j} . \mathcal{N} \Rightarrow t_{i}, t_{j} \in r_{c} . T\right)
\end{aligned}
$$

Aggregation Rule 2 (Copy Relevant Dataflow Transformation Specification of Copied ELEMENTS). The variable and port domains, variable initial values, expected input tokens, and method and transitions side-effects must be copied for the respective copied element.

Aggregation Rule 3 (Extend Invariants of Transitions/Methods). The dataflow transformation specification of a composed partial actor must be extended to include in all allowed side-effects of transitions $T$ and methods $\mathcal{M}$ originating from an actor source $r_{1}$ the specification to leave all variables and output ports originating from the other source actor $r_{2}$ without modification, i.e., invariant.

Aggregation rule 3 ensures that further compositions are possible as every method has a complete description on the side-effects produced on all variables and ports of the composed actor.

4.1.3 Mapping Rules. States, variables and ports of two partial actors can be mapped/merged together by matching ids:

Mapping Rule 1 (Merge Elements By ID). Any two states $s_{i} \in r_{1} . S, s_{j} \in r_{2} . S$, variables $v_{i} \in$ $r_{1} \cdot \mathcal{V}, v_{j} \in r_{2} \cdot \mathcal{V}$, and/or ports $p_{i} \in r_{1} . P, p_{j} \in r_{2} . P$ will be merged from two actor sources $r_{1}$ and $r_{2}$ if they share the same id $\mathcal{N}$ to be used in the merged element of the resulting actor $r_{c}$.

$$
\begin{array}{r}
\forall s_{i} \forall s_{j}\left(s_{i} \in r_{1} \cdot S \wedge s_{j} \in r_{2} \cdot S \wedge s_{i} \cdot \mathcal{N}=s_{j} . \mathcal{N} \Rightarrow \exists s_{c}\left(s_{c} \in r_{c} \cdot S \wedge s_{c} \cdot \mathcal{N}=s_{i} \cdot \mathcal{N}=s_{j} \cdot \mathcal{N}\right)\right) \\
\forall v_{i} \forall v_{j}\left(v_{i} \in r_{1} \cdot \mathcal{V} \wedge v_{j} \in r_{2} \cdot \mathcal{V} \wedge v_{i} \cdot \mathcal{N}=v_{j} \cdot \mathcal{N} \Rightarrow \exists v_{c}\left(v_{c} \in r_{c} \cdot \mathcal{V} \wedge v_{c} \cdot \mathcal{N}=v_{i} \cdot \mathcal{N}=v_{j} \cdot \mathcal{N}\right)\right) \\
\forall p_{i} \forall p_{j}\left(p_{i} \in r_{1} \cdot P \wedge p_{j} \in r_{2} \cdot P \wedge v_{i} \cdot \mathcal{N}=p_{j} \cdot \mathcal{N} \Rightarrow \exists p_{c}\left(p_{c} \in r_{c} \cdot P \wedge p_{c} \cdot \mathcal{N}=p_{i} \cdot \mathcal{N}=p_{j} \cdot \mathcal{N}\right)\right) .
\end{array}
$$

Note that Mapping Rule 1 only considers the id of the merged element, not their valid domain and ranges. So it cannot be concluded that merging by id is always a valid operation.

Also note that in contrast to states, variables, and ports, the methods and transitions of a partial actor are not allowed to be merged but only to be replaced.

Mapping Rule 2 (Replace Methods/Transitions). A method $m_{i}$ or transition $t_{i}$ can be replaced by another method $m_{j}$ or transition ${ }^{10} t_{j}$ if the new method or transition has the same side-effects: (1) Modify the same output variables, (2) produce tokens to the same set of output ports, (3) consume tokens from the same set of input ports, (4) the possible values to be assigned to all variables and output ports are compatible, and (5) the set of possible values of the input variables and input ports is the same.

For example, a method computing the sine function on the variable $x$ and producing its output as a token at port $0 *$ could be replaced with a more accurate implementation, as long as it also takes $x$ as the input parameter, and the output is also produced as a token at port $o *$. The domain of possible

\footnotetext{
${ }^{10}$ Including non-atomic transitions, where this rules applies to all the chains of transitions inside the non-atomic transition originating from the source to the destination state.
} 
values for the output port in this case are compatible, as both sine function implementations should return a value between 0 and 1 . If the new method would write to a different variable or port, or produce a value with a different domain, then a replacement would be invalid.

4.1.4 Rules to Verify Valid Compositions. To verify that the composition of partial actors is valid, the compatibility of merged variables, ports and states need to be checked.

The merging of states requires to verify the incoming and outgoing transitions side-effects, so that the resulting state label is compatible with all the transitions.

Verification Rule 1 (Merged States' Compatibility). At each merged state s, the valid variable domain $V$ and the valid port domain $P E$ of each merged variable $v$, as specified in the resulting state labels of the partial actor sources, must be either equal, or if one of them is a superset of the other, it must be possible to replace the transition-methods side-effects with the superset. ${ }^{11}$

Example: When merging state $S_{B}$ from an actor $r_{1}$ with an FSM: $S_{A} \rightarrow S_{B}$ and state $S_{C}$ from an actor $r_{2}$ with an FSM: $S_{C} \rightarrow S_{D}$, where the valid domain of $S_{C}$ 's variable $v$ is a subset of $S_{B}$ 's, i.e., $S_{C} \cdot \lambda . v \cdot V \subset S_{B} \cdot \lambda \cdot v \cdot V$, then $S_{C} \cdot \lambda \cdot v \cdot V$ becomes $S_{B} \cdot \lambda \cdot v \cdot V$, as long as $S_{C}$ allows it by all outgoing transitions constraints. In this case, a merging is only valid if the transition $S_{C} \rightarrow S_{D}$ accepts the superset $S_{B} \cdot \lambda \cdot v \cdot V$ as the new domain for $v$.

Verification Rule 2 (Merged Variables Compatibility). (1) The variable domains must be equal or, if not, it must be shown that all methods and transitions referencing the variable accept the other domain. (2) The initial value of each merged variable must be consistent in both partial actor sources, i.e., either equal or not specified on at least one of them.

Verification Rule 3 (Merged Ports Compatibility). Valid port domains must be equal, or if a replacement is needed, it must be shown that all other constraints are satisfied with the change, i.e., all methods and transitions referencing the variable accept the replacement domain.

The next rule, ensures the composition of actors that lack isolated states:

Verification Rule 4 (State's Connectedness). Each state $s$ of the composed actor, must not remain isolated, i.e., all states from the resulting actor $r_{c}$ must be part of a single connected graph.

Note that the expected token arrivals specified on partial actor states cannot be verified until a final actor is instantiated, as a partial actor can always be further composed, so the transition originating the token expectation might not yet be part of the partial actor.

Verification Rule 5 (Expected Token Arrival). Only a final actor, as in Definition 3.5, can be verified to always match all state's expected token arrivals defined in $\eta$ to the side-effects of a previous transitions producing expected token arrivals.

4.1.5 Compact Description Operations. To make the specification of partial actor compositions less verbose, the following operations are defined:

Definition 4.1 (Element Addition). The addition of elements to an existing partial actor is defined exactly as if the elements would have been defined in the original creation of the partial actor.

The purpose of the explicit inclusion of addition is to avoid the need to create a separate partial actor that includes a very small set of elements necessary to adapt an existing one for composition.

\footnotetext{
${ }^{11}$ This creates a chain of verification steps through the next state of each transition.
} 


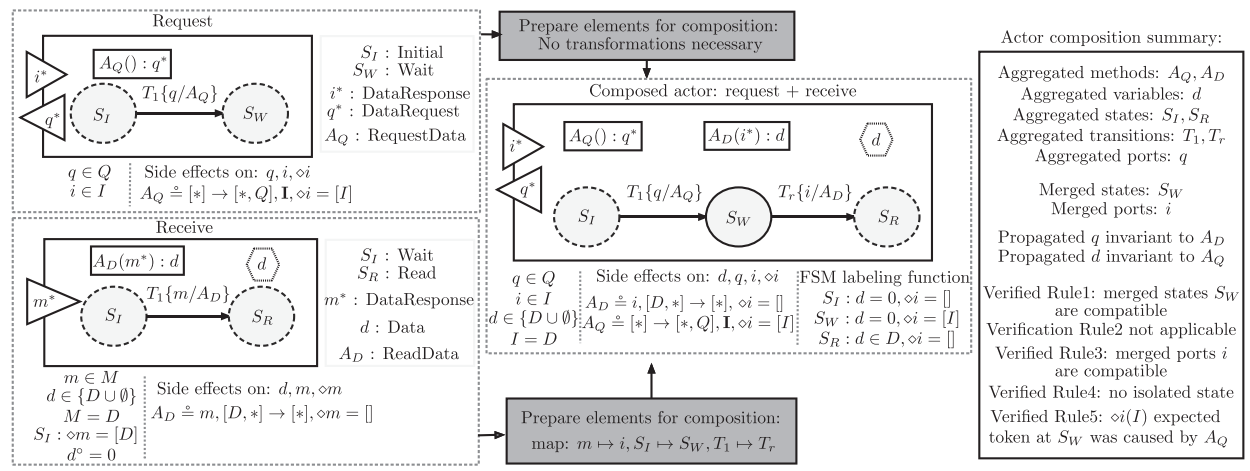

Fig. 4. Example of a valid composition of two partial actors (request + response). The "receive" actor is prepared before composition to match the ids of the "request" actor.

Definition 4.2 (Rename Map). The renaming operations can be specified by a mapping script, providing the new ids of each element from the source partial actors. Furthermore, a set of mappings can be specified to be performed in a single step with \{\} , to avoid cyclic re-mappings. e.g., $S 0 \rightarrow S 1$ can be remaped as $S 1 \rightarrow S 0$ by the following specification: map:\{S0=:S1, $S 1=: S 0\}$.

The following rule simplifies the composition of multiple instances of the same partial actor:

Definition 4.3 (Multiple Instantiation). $N$ multiple instances of a partial actor $r^{p}$ can be used for composition in a single step by (1) adding once each method, variable, and port of the partial actor $r^{p}$ and (2) adding $N$ multiple copies of each state and transition of $r^{p}$ and renaming them by adding to each instance an index suffix to their id.

An example of a valid composition of two partial actors using the rules introduced in this section is shown in Figure 4.

Let us emphasize that the compositional rules presented in this section allow us to analyze a valid syntactical composition of actors, and this composition will often result in an increase in the number of actor elements and thus in the analysis complexity. It is, however, important to highlight that the manual design of a single actor with the same number of elements would not only imply a result with the same size complexity, but will also potentially introduce multiple error-prone design pitfalls compared to a compositional approach of smaller and simpler to understand designs. Techniques to further reduce the analysis complexity, e.g., by compositional analysis techniques, are left out-of-the-scope of this work.

\section{STATE-OF-THE-ART}

This section provides a reference to existing time-aware modeling languages and methodologies for the specification of CPS, as well as a summary on relevant design patterns and provides arguments for the need of a modeling paradigm to express and compose reusable QoI-aware design patterns.

\subsection{Modeling of Real-time CPS}

In the following, the most relevant modeling paradigms are compared to our approach.

\subsubsection{Real-time Modeling Languages.}

Realtime Java [11]: This runtime library provides a deterministic garbage collection and scheduler to support real-time software. Strengths: wide adoption and high logic reusability of 
object-oriented design. However, OO designs are difficult to scale in multi-threading correctly, and it only supports the platform-dependent asynchronous model of time.

SDL [45]: This is a standard for telecom specifications, which abstracts away system implementation. It defines behavior of components through extended FSMs and allows hierarchical designs. The communication semantics between components is asynchronous and is equivalent to an unbounded FIFO channel. Provides the concept of timers to monitor durations. Strengths: It can describe complex behaviors and is broadly adopted. However, logic reuse is difficult. TimedSvFunState comparison: Messages received are silently deleted if not expected, and thus the specification of synchronous communication (blocking) is not possible. Action durations necessary for the preestimated time model can only be included through an extension called SDL* [46].

Esterel [7], Lustre [23], and Signal [21]: These are synchronous languages, where Esterel is imperative that defines explicit sequence of statements and the other two declarative and use equations expressing functional or relational dependencies. Strengths: suited for defining platform independent functionality where time has been abstracted away. TimedSvFunState comparison: It can only use a single global clock and cannot model implementation details.

Statecharts [24]: This is an imperative language: defines explicit sequence of statements. Strengths: allows for hierarchical designs. Weaknesses: it can be interpreted with different possible semantics, and logic reusability is low. TimedSvFunState comparison: uses shared memory instead of message passing, which makes difficult the design of scalable concurrent systems.

Giotto [25]: Communication is instantaneous, and computation is scheduled. Strengths: supports multiple clocks and the modeling of reactive processes. Supports the preestimated time model for verification. Separates platform dependent from independent concerns through annotations TimedSvFunState comparison: it is limited to periodic tasks.

FSMs: The Finite State Machine formalism is well suited to describe control behaviors being thus very relevant for CPS. The two of the most relevant variations for this work are as follows:

Timed automata [1]: It is the automata formalism extended with real valued variables called "clocks" that can be used as timers. UPPAAL [32] and KeYmaera [37] are verification tools that provide support for this formalism. Strengths: good for verification due to the preestimated time model. Weaknesses: a single global clock and low reusability. Only a restricted subset of the target formalism of the proposed work could be transformed to the timed automata formalism for the integration to the related verification tools. Concretely, the MoC must be constrained to a preestimated time model, i.e., only use static annotations of time, as well as the usage of guard and actions that can be described by mathematical formulas.

Extended FSMs (EFSMs): These include variables to further specify the internal state to create more complex designs with significantly less states but with increased analyzability complexity.

However, the reusability of FSM-based behavior description is a difficult task. Control flow logic specified by FSMs is difficult to reuse due to the context-dependency of its elements. The specification of states and transitions conveys a lot of context information. For example, transitions can only be reused in another FSM if all of their assumptions in their current source state are also satisfied in the new FSM. These assumptions may refer to side-effects inside local variables in EFSMs and/or side-effects produced to the environment of FSMs.

Interface automata $[13,34]$ provides an approach to address reusability of FSMs by allowing the specification of the sequence of events of a process (i.e., addresses the logical time) and their composition. It defines a set of acceptable events on each state. Composing two automata means to generate a third automata where each of its new states corresponds to a state in both automata sources. Because it defines an optimistic approach for composing FSMs (FSMs must valid for some environment), any composed state that is not accepted by one of the automata sources is removed, 
and the resulting composed automata is consider valid. Thus, interface automata provides a composable paradigm for FSM specifications.

However, a limitation of the interface automata composition formalism, is that it only considers external events and cannot be used if the MoC allows the use of variables as defined in EFSMs. This reduces the expressability and scalability to define complex specifications.

The presented approach is based on TimedSvFunState to allow specifications in multiple MoCs, including the use of local variables. However, the TimedSvFunState formalism lacks the necessary information to allow valid compositions, as it does not specify what transformations are applied to its local variables and I/O on any of the FSM transition actions. Thus, for reusability purposes, it is not possible to deduce from a specification created in TimedSvFunState if the performed sideeffects by transitions will meet or violate the assumptions of another FSM after composition.

In this work, the TimedSvFunState formalism is extended to enable valid behavior compositions.

In comparison to interface automata, our approach does not restrict FSMs to only "accept" tokens explicitly specified. Any two components are connected via a bounded FIFO channels, and a token then can be consumed asynchronously at a different state at a later stage. Regarding the composition of FSMs, our approach is pessimistic, as FSMs must be compatible for all environments, where the meaning of compatible will be provided in Section 4.

5.1.2 Model-based Design. None of the realtime modeling languages can be used alone to jointly specify the behavior of the heterogeneous components of CPS, provide reusability, and model the effects of system implementation. Model-based system-level methodologies have been proposed as a solution:

UML-based: Applicable in OO design, several methodologies based on UML have been proposed to include the modeling of the system implementation (MARTE [36], UML-RT [42], and Real time UML [22]). Behavior specification is allowed through activity diagrams, FSMs, or sequence diagrams. They also extend UML to allow the definition of annotations of nonfunctional properties.

Simulink [35]: Tool for dataflow actor-oriented modeling that provides a big library for graphical component-based design. Physical (continuous) time is advanced in parameterizable steps.

PtolemyII [16]: Provides an actor-oriented framework to explore the modeling of CPS under different MoCs through the separation of dataflow and control flow through so-called domain directors. It supports aspect-orientation to evaluate different implementations based on the Y-Chart [29] approach. Strengths: inter-actor composability of multiple time semantics.

MAESTRO [38, 39]: An actor-oriented framework for the modeling of functionality, implementation, and nonfunctional property evaluators. Its time semantics are defined by the TimedSvFunState. Strengths: enables the hierarchical design of CPS in a single specification language and enables the modeling of interdependent nonfunctional properties for dynamic simulation-based evaluation.

AADL $[18,41]$ : Provides a declarative framework to define CPS specifications and models of their implementation. Through the behavior annex extension, complex behaviors can be specified through FSMs. Strengths: declarative, analyzable (e.g., scheduling, reliability).

Intel CoFluent [10]: Provides a GUI front-end to model system specifications based on activity diagrams that can generate SystemC simulation code.

The presented approach can be implemented in PtolemyII, MAestro, AADL, or any other methodology that could support the TimedSvFunState MoC.

\subsection{Design Patterns for Real-time and Safety-Critical Embedded Systems}

Many design patterns have been identified in real-time systems to specify concurrency, architecture, resource sharing, and distributed systems using object-orientated UML [15]: 
Watchdog Timers [15] and local clocks are usually used to monitor time window constraints of computation and communication processes.

Reactive processes. One alternative to scheduled processes bounded by time windows in realtime systems design, is the specification of reactive processes through the separation of the interface process to handle environment events and the execution process scheduling (blocking) tasks. This pattern is employed in Giotto [25], as it separately specifies a driver engine and an execution engine (called embedded machine and scheduling machine, respectively) [26].

Safety-critical systems must employ a repertoire of dependability [6] increasing techniques to minimize and bound risk to acceptable levels. Diversity is often used to implement multiple redundant channels components of systems that can be used to increase availability, reliability or safety. The following is a set of a few design patterns [2] that exploit diversity in different ways:

M-Out-Of-N Pattern (M-oo-N): Uses N redundant homogeneous (identical) channels to perform a process that run concurrently. $\mathrm{M}$ components are at least required to agree on their outcome, from which a majority voter selects as valid output. It enhances system reliability in the presence of random faults.

Monitor-Actuator [15]: Special kind of M-oo-N. Defines one actuation and one monitoring channel to detect and identify possible faults on the actuation channel to drive it to a safe state.

N-Version Programming (NVP) [5, 9] (a.k.a Master-Slave Pattern): This is a variation of the $\mathrm{M}$-oo-N pattern that defines the creation of $\mathrm{N}>=2$ different implementations of the original specification that will be executed concurrently and voted for. It addresses systematic design errors. Has been originally defined for fault-tolerant SW, so heteregeneous HW resources should be considered for systematic error robustness.

Recovery Block (RB) [27]: Similar to NVP but executes a single implementation at a time, defining an acceptance test to determine a successful process execution. If the test fails, then another implementation is executed.

Many more patterns exist that combine spatial, temporal and implementation diversity, such as Acceptance Voting [4] and Recovery Block with Backup Voting [3].

In actor-oriented design, there exists little references on how to define similar patterns in semantics different than OO, where control flow and dataflow are not separated. Existing solutions, e.g., Reference [40], provide design patterns that aim to modularize pieces of a behavior specification in separate actors (e.g., each with its own FSM), whereas the proposed approach defines more granular design patterns for the composition of a single actor behavior specifications.

To the best of our knowledge, there is no equivalent comprehensive set of design patterns in extended FSM models of computation as in the gang of four in object-oriented design [20] for the creation of CPS behavior with awareness of several QoI properties.

\section{DESIGN PATTERNS FOR QUALITY-OF-INFORMATION-AWARE CPS}

In this section, five reusable design patterns, one for each QoI property, are presented in the proposed approach. Each pattern describes the problem addressed and related patterns and provides a control flow template that can be composed and customized to different contexts. These patterns are not meant to cover all possible behaviors related to QoI but to illustrate the composable approach to jointly address them.

\subsection{Timeliness: Time Window Monitor}

Problem. Identify violations of a response's time window constraints in a request-response handshake, and branch the control flow according to three categories: Early, On-time, and Late. 


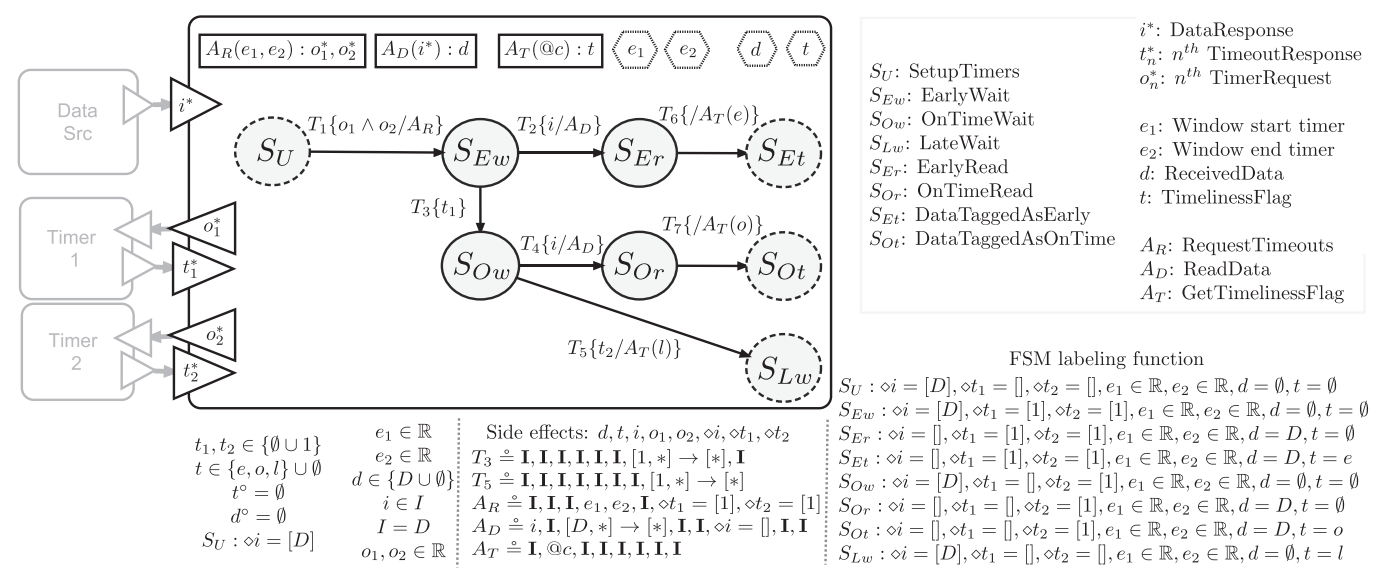

Fig. 5. Time window monitor: Two timers are used to branch the control flow when waiting to received data.

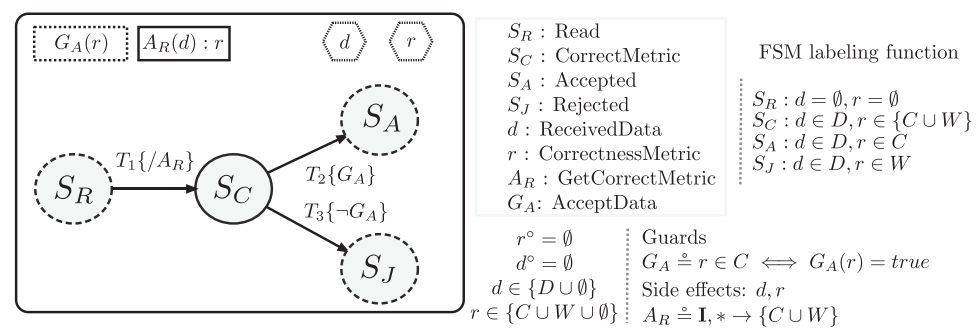

Fig. 6. Branch on error: Split the control flow according to some correctness metric.

Related design patterns. Watchdog Timers [15] for branching on a late response, and The hare and tortoise [40] pattern describing a similar but not composable approach.

Solution. Figure 5: Assumes a token response is expected from a data source at port $i^{*}$ due to some data request. Setup two external timers to identify the start and the end of the time window constraint. A tag $t$ (a local variable) is set to indicate the reception of data according to the order of the token arrival events: Early: $e$ if $i . \tau<t 1 . \tau$ On-time: $o$ if $t 1 . \tau<i . \tau<t 2 . \tau$ Late: $l$ if $t 2 . \tau<i . \tau$.

\subsection{Correctness: Branch on Error}

Problem. Binary categorization of data based on a correctness metric and branch control flow.

Related design patterns. General error detection paradigms such as return codes, forward error correction. Allows to implement the "Let-it-crash" pattern [30] to move to a system reset state that is more cost effective than to handle the local error.

Solution. Figure 6: Assumes data were already acquired. Executes an oracle function to evaluate the data correctness $r$. Branches in two, according to a guard $G_{-} A$ as a function of the metric $r \in R \cup W$.

\subsection{Completeness: Request to Complement Incomplete Data}

Problem. Identify if there exist known unknowns in the received data to be resolved and request the data source for the missing information. 


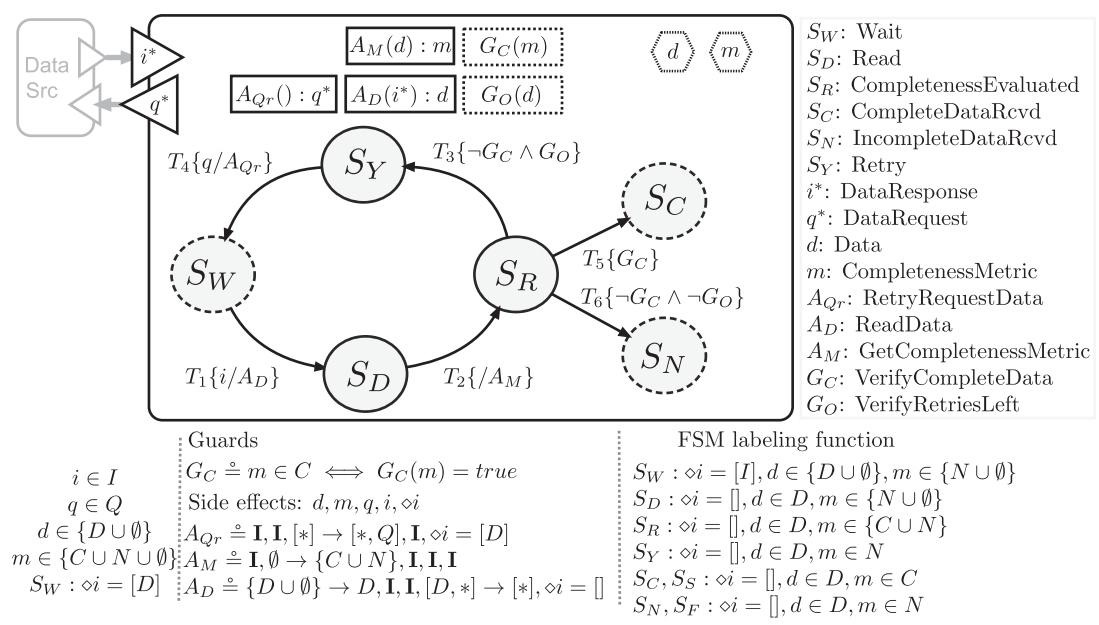

Fig. 7. Request to complement incomplete data: Evaluate data and request complement if needed.

Related design patterns. The Automatic repeat request method, which retransmits information if no acknowledgement of reception is received. This pattern however focuses on the receptor side and evaluates the completeness of each response.

Solution. Figure 7: Assumes a token response is expected from a data source actor at port $i^{*}$ due to some data request. The completeness of the received data is evaluated according to some metric $m \in C \cup N$. The control flow is branched according to a binary categorization of complete $m \in C$ or incomplete $m \in N$. If data are deemed incomplete, then a complementary request is sent to the data source for a maximum number of retries specified by $G_{-} O$.

\subsection{Consistency: Multiple Sources of Data}

Problem. Decide on the most likely value of data obtained from multiple parallel sources or validate the correlation of information aggregated from multiple sources.

Related design patterns. [2]: Dependability increasing design patterns using redundant sources of information: such as NVP, MooN, homogeneous, heterogeneous, with/without diagnostics, and so on.

Multiple sources in time: Special case of the combination of this pattern with one single source plus the correctness pattern.

Solution. Figure 8: Request and gather data from multiple parallel sources of information. Once all sources have responded, the consistency metric $c$ is evaluated. Note that the number of variables, ports and transitions reading them is dependent on the number of parallel sources required.

\subsection{Accuracy: Iterative Data Accuracy Improvement from Multiple Sources}

Problem. Improve accuracy of data from different data sources (in space or time)

Related design patterns. A Kalman filter can be implemented with this pattern.

Solution. Figure 9: Request data and estimated accuracy from multiple sources, and perform an operation (e.g., update step) on received data. Provide data to clients and allow to interrupt and exit. 


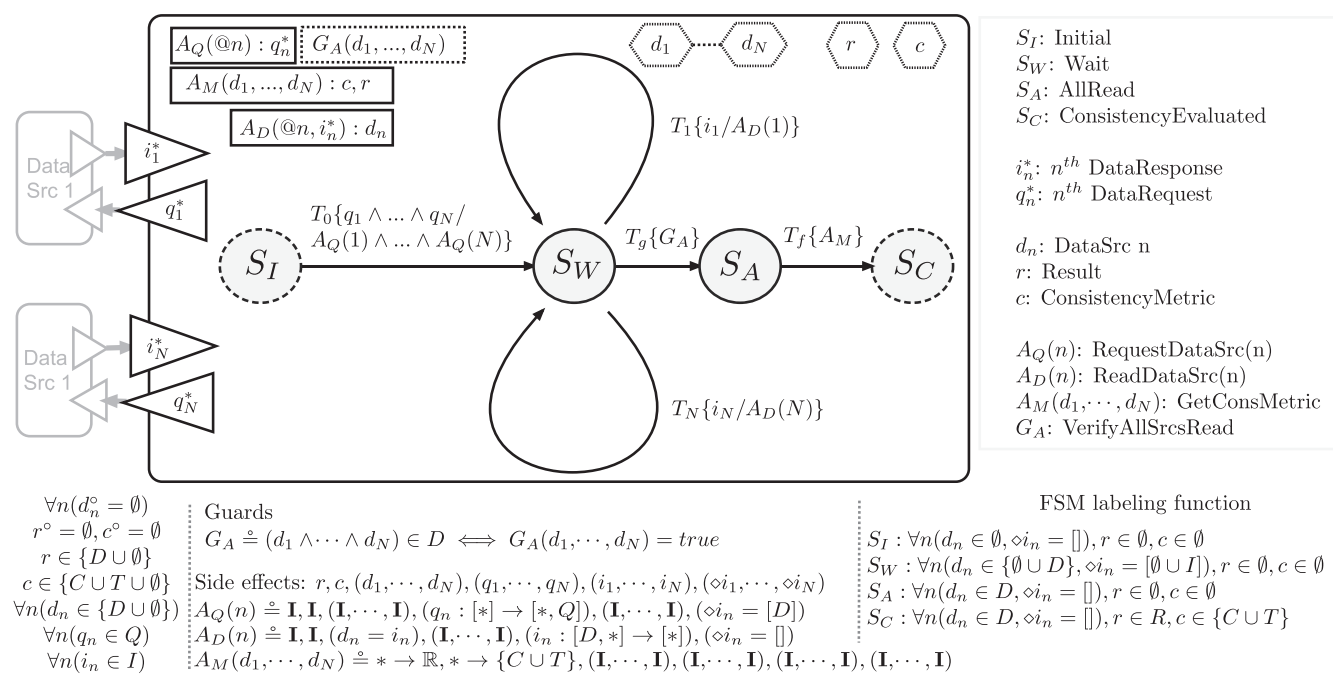

Fig. 8. Multiple sources of data: Wait for data from $N$ sources (shown $N=2$ ) and evaluate their consistency.

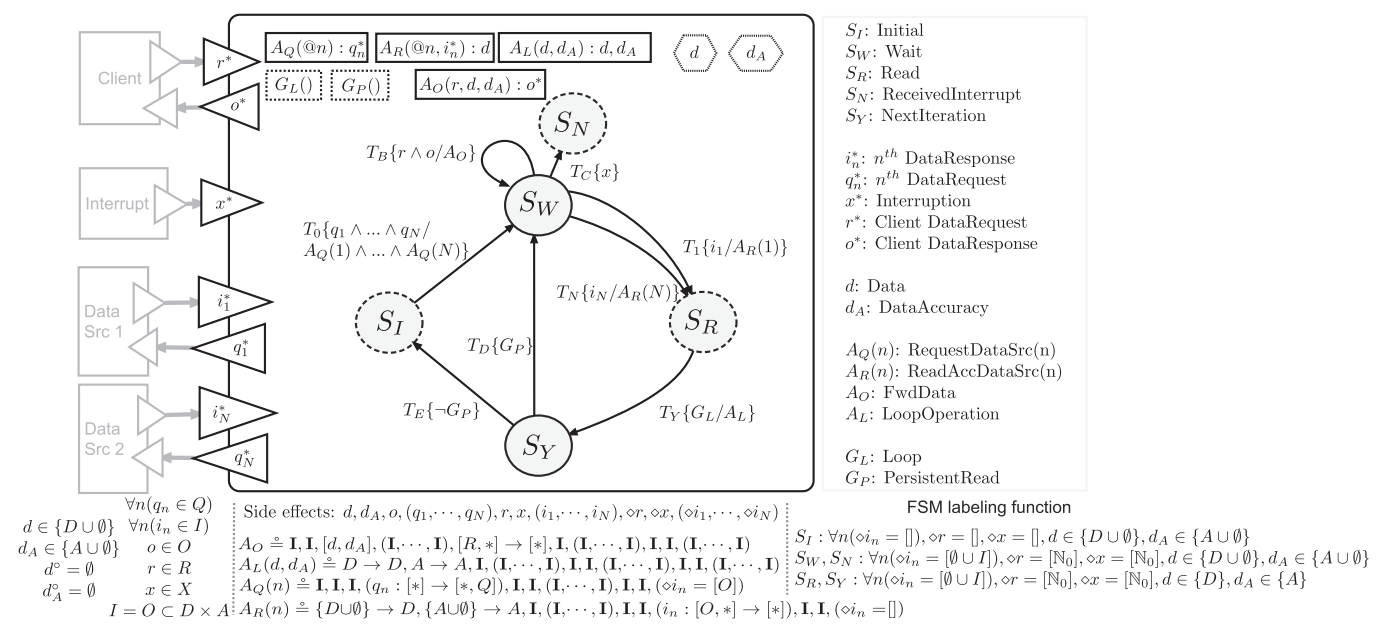

Fig. 9. Improve accuracy iteratively from multiple sources.

\section{MODEL-DRIVEN COMPOSITION OF QOI-AWARE CPS DESIGN PATTERNS}

The time to design CPS behavior that considers more than one QoI can be reduced by combining design patterns using the composition rules of Section 4 . The design time improvement is achieved by splitting the design of a complex system in smaller and reusable design patterns that can be combined in a model-driven approach.

To compactly describe five different compositions of timeliness with other QoI design patterns, a graphical representation of the state's semantics is first introduced in Figure 10. Instead of textually describe each state label, different visual elements are used to reflect the QoI metrics of data at each state, as well as a different representation of the transitions according to the performed operation.

Figure 11 show the FSMs of the five design patterns using the introduced symbolic notation for the state's labels and for their relevant transitions. 


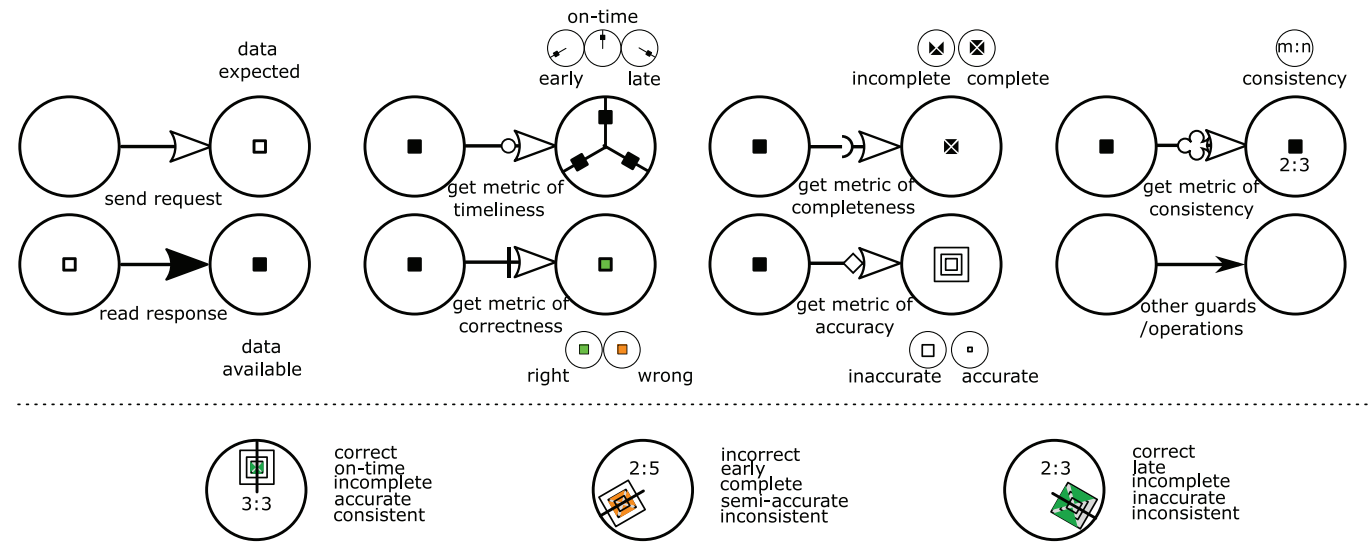

Fig. 10. Symbolic notation of state's and transition's semantics. Top: The states labels with the evaluated Qol metrics are represented graphically through different visual degrees of freedom: data availability with a token, timeliness depicted with the angle, correcteness with color, completeness with slices of the token, accuracy with the token size, and consistency with a text label. Bottom: Three examples with all metrics.

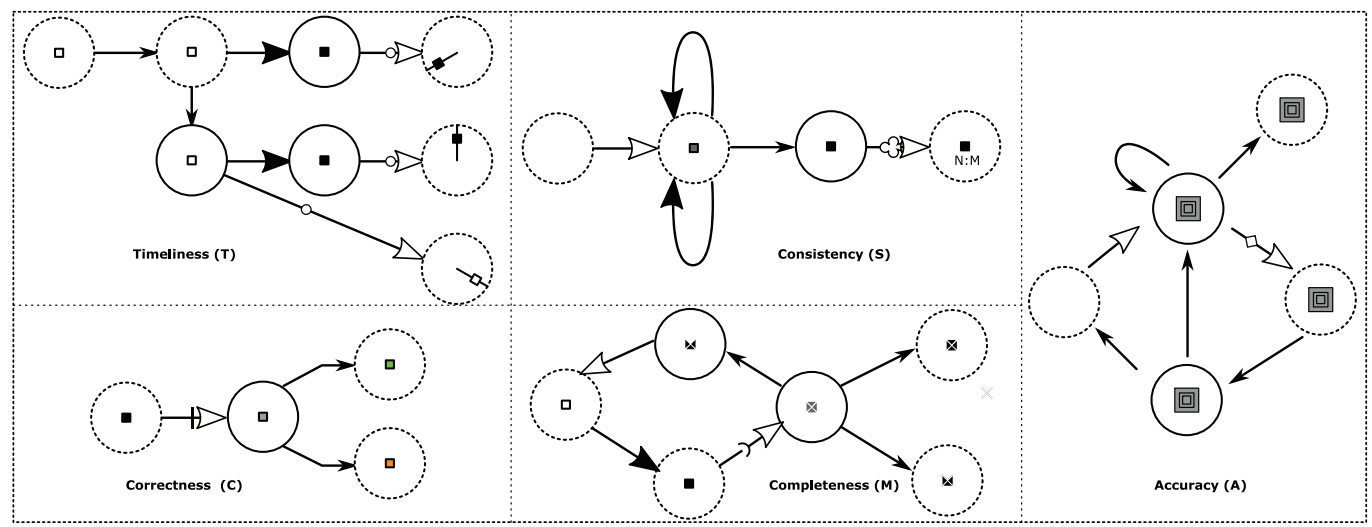

Fig. 11. Graphical representation of the FSM of the five patterns using the diagram notation to easily identify state and transition semantics to perform design pattern's composition visually.

In Figure 12, five different compositions of the timelines pattern are shown. The transformation rules are omitted, as well as the ports, actions, variables, and connection to external actors (such as timers). The symbolic notation, however, enables to identify the compatibility of states when composing each pattern.

For example, in the composition of timeliness and correctness on the top left, the branch of control flow where the time window has expired does not have data available, thus to merge this branch with the other two, an intermediate step to wait for the data is necessary. This can be visualized by the hollow token that needs to be filled at the receive pattern.

Different possible values may result from an evaluation of a QoI property, so only after a guarded transition splits the control flow it is possible to uniquely identify a state to a specific value of a property. See for example the correctness pattern where the colors are well defined after a metricbased branch. 

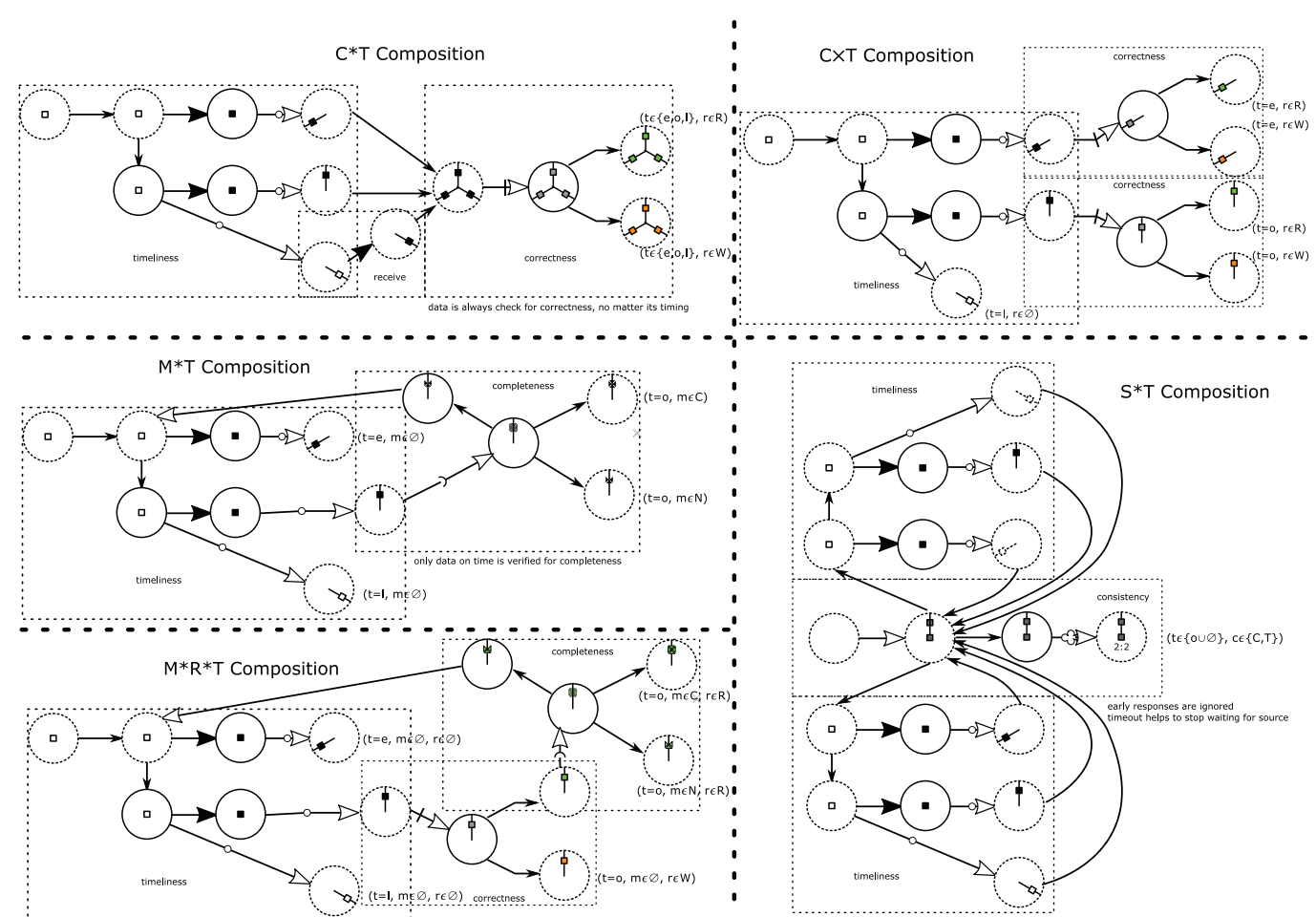

Fig. 12. Example compositions of timeliness. Top: Timeliness plus Correctness. The $\mathrm{C}^{*} \mathrm{~T}$ composition requires the addition of a receive step to wait for late data. The CxT composition verifies the correctness of each branch independently and thus the final states are always specific to each combination of the two metrics. Bottom left: Timeliness plus correctness plus completeness. If correct data received on time is not complete, then a respective request for the missing data is made within the current time window. Bottom right: Timeliness plus consistency. Each parallel data source is checked for timeliness, where early and late responses would not take part in a voting operation.

Note that the patterns can be composed multiple times and in different ways. The shown compositions are only meant to illustrate the possibilities and demonstrate the proposed approach to develop composable partial specifications.

\section{CASE STUDY: QOI-AWARE INFRASTRUCTURE-ASSISTED C-AD}

As a case study, an executable specification of a vehicle's sensing application subscribed to receive environment information from the roadside infrastructure to enhance its ego perception is created. This specification is evaluated against two different V2I architecture implementations using either the LTE-Uu cellular network or a direct sidelink interface.

The proposed model-driven approach has been used to code and reuse the QoI design patterns to create the functionality specification of the sensing application so that the vehicle can behave accordingly to the limitations at the road affecting the received QoI.

The fusion of the sensor data originating from the roadside infrastructure with the vehicle's own sensor information requires to (1) synchronize both systems and (2) validate the exchanged data in several aspects, e.g., how fresh it is, source authentication, or transmission errors.

Figure 13 shows the modeled case study of the vehicle behavior with the proposed design patterns. After synchronization hand-shakes, the timeliness pattern of the time window is used to 


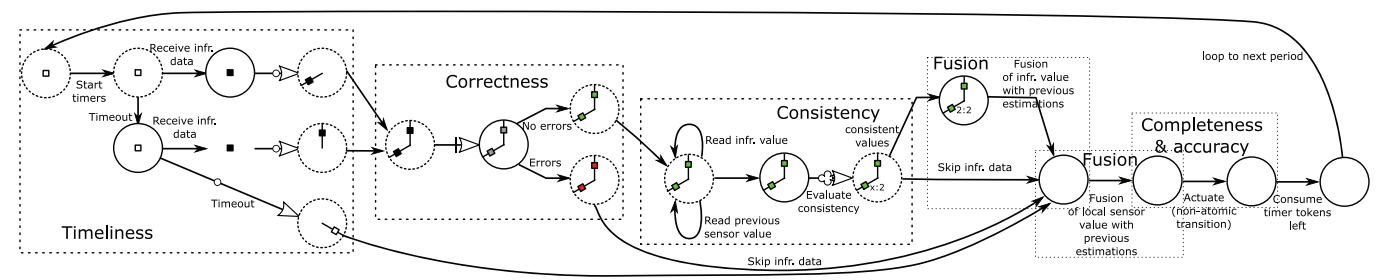

Fig. 13. Vehicle behavior created from composed design patterns to monitor the timeliness, correctness, and consistency of sensor data sent from the infrastructure to the vehicle. This specification fuses the infrastructure sensor data if the aforementioned Qol properties are adequate and allows us to refine the actuation step with a non-atomic transition that can be replaced with different accuracy and completeness monitors.

wait for a limited amount of time for infrastructure data. If data are received on the agreed-upon scheduled time window, then they are first verified for correctness and then fused with the vehicle's own model. In case it is not received on time, the vehicle proceeds to update its environmental model using only its own sensors. Correct messages received before or during the time window trigger the evaluation of their consistency to verify that delayed data is still consistent with the vehicle perception.

API snippet for the composition example of Figure 4

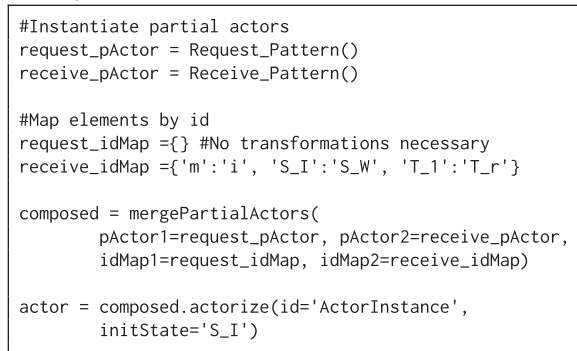

The modeling of the executable specification was done via a proprietary software library in Python for the model-driven creation and composition of the design patterns complying with the transformation rules. See left for a small snippet of the API. The output is a scripted description of partial actors in the TimedSvFunState MoC, which is then used to create an actor network as an executable specification in a semantically equivalent language to the open source SysteMoC.

A scenario of $5 \mathrm{~s}$ of communication between the infrastructure and a vehicle was simulated. The scenario consists of both the infrastructure and the vehicle perceiving the presence of a slowly taking-over vehicle with constant speed. Three different simulation runs are performed in this scenario, to test the algorithm performance when I2V communications according to roadside availability are scheduled every $10 \mathrm{~ms}$ through (a) a side link communication with a latency much smaller than the listening period, (b) the cellular network link with a Poisson distributed probability to incur penalty delays $(20$ to $200 \mathrm{~ms}) 3 \%$ of the time. In the second case, two different experiments are performed: (1) Such delayed messages are always fused into the vehicle's model, and (2) depending on the consistency check, some delayed messages are fused.

The modeling of both architecture implementations was done at the electronic system level of abstraction, i.e., real functionality of the communication protocol stack is abstracted away and a a preestimated model of time is used to evaluate the timing of actions. ${ }^{12}$ For the modeling of the sensors and data fusion, a Kalman filter and the Covariance Intersection algorithm were applied using a constant velocity model to validate the demonstrator of the proposed approach. The modeling time of the case study using the model-driven approach was $\sim 4$ person's days. One day was invested in the individual design patterns, one day in their composition and 2 days

\footnotetext{
${ }^{12}$ Fine-grained evaluations considering real functionality are also possible but usually required at later design stages.
} 

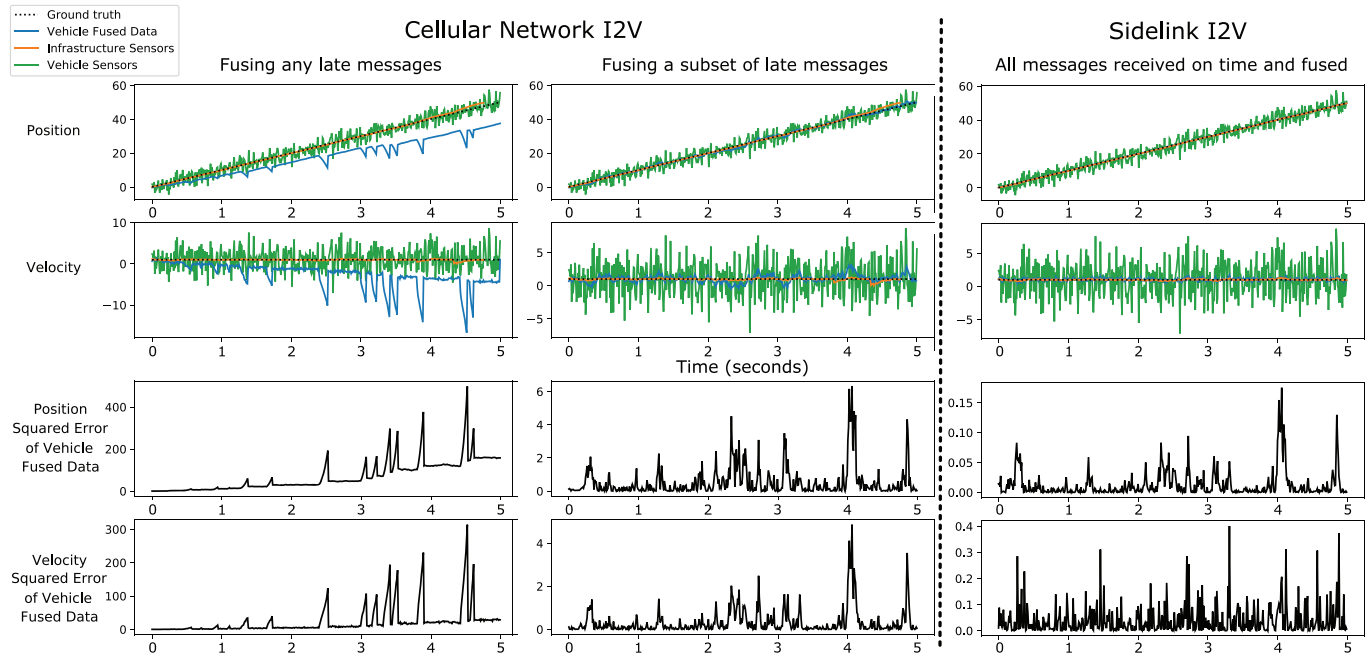

Fig. 14. Three scenarios for fusion of infrastructure data into vehicle's environment model. Right: A Sidelink communication link is used where every message arrives within the expected window, providing the lowest estimation error. Left: $12 \mathrm{~V}$ communications through the cellular network. If every delayed message is blindy accepted, then the fusion algorithm begins to diverge. If the delayed messages are selectively filtered, then the vehicle's estimation error can be improved.

in the implementation of the fusion algorithm actions. The proposed approach thus allowed to define in one day the actor-oriented specification of QoI property monitor logic once the design patterns have been defined. Compared to the equivalent time needed to model the same actor-oriented specification without this approach provides already a reduction of design-time by half even though this case study does not instantiate the design patterns multiple times, as would be case in the specification of a communication protocol for example. The design pattern in the source language are specified on average on 70 Lines of Code $(\mathrm{LoC})$. The average number of LoC necessary to compose two partial actors was approximately 20, including the name remappings. The total execution time of each experiment was on average $35 \mathrm{~s}$ (mostly consumed on data fusion actions) on a standard personal computing setup.

Figure 14 shows the obtained data fusion results in the three experiments. The low-latency sidelink communications with no data fusion problems and the cellular-network in two configurations. The first at the left fuses all delayed messages into the vehicle's env. model, which results in an accumulation of the estimation error. The middle cases shows the filtering of late messages achieving a better result for this configuration. The three performed experiments attest that the sensing algorithm should not be designed to ignore the timeliness of the received information, and that the design of QoI-aware algorithms is necessary for the development of robust CPS.

The main benefits of the model-driven approach demonstrated by the case study can be summarized as follows: (a) Reduced modeling effort: The total lines of code needed for creating the specification in the model-driven approach are reduced from $\sim 210$ to $\sim 60$ for the composition of patterns. This translates to less effort and time dedicated in implementing the handling of QoI properties. (b) Robust specifications: The case study shows that by monitoring the QoI properties, the estimation error in the vehicle data fusion algorithm can be controlled in system implementations with variations from ideal conditions, in this case for latency. (c) Dynamic evaluation of time: The model timing can be evaluated dynamically in simulation, enabling to validate the model response to timing variations due to system implementation diversity and/or environment conditions. 


\section{CONCLUSION}

We have presented a formalism to enable the definition of composable EFSM behavior specifications. The proposed model-driven approach allows us to specify reusable control flow design patterns that can be used to jointly address QoI properties such as timeliness in CPS.

The benefits of the model-driven approach based on design patterns in the TimedSvFunState $\mathrm{MoC}$ are (1) a reduced modeling effort by the composition of simple EFSMs-based behaviors and reduce modeling errors through the reuse of solution recipes, (2) combinations of timeliness and other QoI properties can be managed in CPS for complete system behavior descriptions, and (3) CPS specifications can be validated against different implementations and non ideal scenarios with existing ESL methodologies in the preestimated, asynchronous and synchronous time models.

The presented work could be extended to combine formal verification techniques with the proposed specification formalism, possibly as described in Section 5.1.1. Furthermore, other design patterns can be developed to address different QoI, QoS, safety, and/or security-aware behaviors.

\section{REFERENCES}

[1] Rajeev Alur and David L. Dill. 1994. A theory of timed automata. Theor. Comput. Sci. 126, 2 (1994), 183-235. DOI : https://doi.org/10.1016/0304-3975(94)90010-8

[2] Ashraf Armoush. 2010. Design Patterns for Safety-critical Embedded Systems. Ph.D. dissertation. RWTH Aachen University.

[3] Ashraf Armoush et al. 2008. A hybrid fault tolerance method for recovery block with a weak acceptance test. In Proceedings of the IEEE/IPIP International Conference on Embedded and Ubiquitous Computing, Volume I. 484-491.

[4] Ajay Athavale. 1990. Performance Evaluation of Hybrid Voting Schemes. Ph.D. dissertation. North Carolina State University.

[5] Algirdas Avizienis. 1985. The N-version approach to fault-tolerant software. IEEE Trans. Sofw. Eng. 11, 12 (1985), 1491-1501.

[6] Algirdas Avizienis, Jean-Claude Laprie, Brian Randell, and Carl E. Landwehr. 2004. Basic concepts and taxonomy of dependable and secure computing. IEEE Trans. Depend. Sec. Comput. 1, 1 (2004), 11-33.

[7] Gérard Berry and Laurent Cosserat. 1984. The ESTEREL synchronous programming language and its mathematical semantics. In Proceedings of the International Conference on Concurrency. Springer, 389-448.

[8] Michael C. Browne, Edmund M. Clarke, and Orna Grumberg. 1988. Characterizing finite kripke structures in propositional temporal logic. Theor. Comput. Sci. 59, 1-2 (1988), 115-131. DOI : https://doi.org/10.1016/0304-3975(88)90098-9

[9] Liming Chen and Algirdas Avizienis. 1977. On the implementation of n-version programming for software fault tolerance during program execution. In Proceedings of the International Computer Software and Applications Conference (COMPSAC'77).

[10] Intel Corporation. 2020. Intel CoFluent Technology. Retrieved March 2, 2020 from https://www.intel.com/content/ www/us/en/cofluent/overview.html.

[11] TimeSys Corporation. 2006. RTSJ: The Real Time Specification for Java 1.0.2. Retrieved March 18, 2019 from https://jcp.org/aboutJava/communityprocess/mrel/jsr001/index2.html.

[12] James Cremer, Joseph Kearney, and Yiannis Papelis. 1995. HCSM: A framework for behavior and scenario control in virtual environments. ACM Trans. Model. Comput. Simul. 5, 3 (1995), 242-267.

[13] Luca de Alfaro and Thomas A. Henzinger. 2001. Interface automata. In Proceedings of the 8th European Software Engineering Conference. 109-120.

[14] Patricia Derler, Edward A. Lee, and Alberto L. Sangiovanni-Vincentelli. 2012. Modeling cyber-physical systems. Proc. IEEE 100, 1 (2012), 13-28.

[15] Bruce Powel Douglass. 2003. Real-time Design Patterns: Robust Scalable Architecture for Real-time Systems. AddisonWesley.

[16] Johan Eker, Jorn W. Janneck, Edward A. Lee, Jie Liu, Xiaojun Liu, Jozsef Ludvig, Sonia Sachs, Yuhong Xiong, and Stephen Neuendorffer. 2003. Taming heterogeneity-The Ptolemy approach. Proc. IEEE 91, 1 (2003), 127-144.

[17] Joachim Falk, Christian Haubelt, and Jürgen Teich. 2006. Efficient representation and simulation of model-based designs in SystemC. In Proceedings of the Forum on Specification and Design Languages (FDL'06), Vol. 6.

[18] Peter H. Feiler and David P. Gluch. 2012. Model-Based Engineering with AADL-An Introduction to the SAE Architecture Analysis and Design Language. Addison-Wesley.

[19] Abdoulaye Gamatié. 2010. Designing Embedded Systems with the SIGNAL Programming Language-Synchronous, Reactive Specification. Springer. DOI : https://doi.org/10.1007/978-1-4419-0941-1 
[20] Erich Gamma, Richard Helm, Ralph Johnson, and John Vlissides. 1993. Design patterns: Abstraction and reuse of object-oriented design. In Proceedings of the Annual Conference on Object-Oriented Programming (ECOOP'93), Oscar M. Nierstrasz (Ed.). Springer.

[21] Thierry Gautier and Paul Le Guernic. 1987. SIGNAL: A declarative language for synchronous programming of realtime systems. In Proceedings of the Functional Programming Languages and Computer Architecture. 257-277.

[22] Object Management Group et al. 2002. UML Profile for Schedulability, Performance, and Time Specification. Retrieved from http.://www.omg.org/cgibin/doc.

[23] Nicholas Halbwachs, Paul Caspi, Pascal Raymond, and Daniel Pilaud. 1991. The synchronous data flow programming language LUSTRE. Proc. IEEE 79, 9 (1991), 1305-1320.

[24] David Harel. 1987. Statecharts: A visual formalism for complex systems. Sci. Comput. Program. 8, 3 (1987), $231-274$. DOI : https://doi.org/10.1016/0167-6423(87)90035-9

[25] Thomas A. Henzinger, Benjamin Horowitz, and Christoph M. Kirsch. 2001. Giotto: A time-triggered language for embedded programming. In Proceedings of the 1st International Workshop on Embedded Software (EMSOFT'01). 166184.

[26] Thomas A. Henzinger, Christoph M. Kirsch, and Slobodan Matic. 2003. Schedule-carrying code. In Proceedings of the 3rd International Conference on Embedded Software (EMSOFT'03). 241-256.

[27] James J. Horning, Hugh C. Lauer, P. M. Melliar-Smith, and Brian Randell. 1974. A program structure for error detection and recovery. In Proceedings of the International Symposium on Operating Systems. 171-187.

[28] ISO/PAS. 2019. ISO/PAS 21448:2019 Safety of the Intended Functionality. Standard 21448.

[29] Bart Kienhuis, Ed Deprettere, Kees Vissers, and Pieter van der Wolf. 1998. The construction of a retargetable simulator for an architecture template. In Proceedings of the 6th International Workshop on HW/SW Codesign. IEEE Computer Society, 125-129.

[30] Roland Kuhn, Brian Hanafee, and Jamie Allen. 2017. Reactive Design Patterns. Manning Publications Company.

[31] Leslie Lamport. 1994. The temporal logic of actions. ACM Trans. Program. Lang. Syst. 16, 3 (1994), 872-923.

[32] Kim G. Larsen, Paul Pettersson, and Wang Yi. 1997. Uppaal in a nutshell. Int. J. Softw. Tools Technol. Transf. 1, 1 (01 Dec. 1997), 134-152. DOI : https://doi.org/10.1007/s100090050010

[33] Edward A. Lee and Alberto Sangiovanni-Vincentelli. 1996. The tagged signal model-a preliminary version of a denotational framework for comparing models of computation. Departments of EE and CS, University of California. https://ptolemy.berkeley.edu/papers/96/denotational/.

[34] Edward A. Lee and Yuhong Xiong. 2004. A behavioral type system and its application in Ptolemy II. Form. Asp. Comput. 16, 3 (2004), 210-237. DOI : https://doi.org/10.1007/s00165-004-0043-8

[35] Mathworks. [n.d.]. Simulink. Retrieved Mar 2, 2020 from http://www.mathworks.com.

[36] UML OMG. 2011. UML Profile for MARTE: Modelling and Analysis of Real-Time Embedded Systems, Version 1.1. https://www.omg.org/spec/MARTE/1.1/About-MARTE/.

[37] André Platzer and Jan-David Quesel. 2008. KeYmaera: A hybrid theorem prover for hybrid systems (sys. description). In Automated Reasoning, Alessandro Armando, Peter Baumgartner, and Gilles Dowek (Eds.). Springer, 171-178.

[38] Rafael Rosales. 2017. Holistic Actor-Oriented Modeling of Embedded Systems for ESL Power Consumption Evaluation. Ph.D. dissertation. Friedrich-Alexander-Universität Erlangen-Nürnberg.

[39] Rafael Rosales et al. 2014. MAESTRO - Holistic actor-oriented modeling of nonfunctional properties and firmware behavior for MPSoCs. ACM Trans. Des. Autom. Electr. Syst. 19, 3 (2014), 23:1-23:26.

[40] Rafael Rosales, Wael Guibene, and German Fabila Garcia. 2018. Actor-oriented design patterns for performance modeling of wireless communications in cyber-physical systems. In Proceedings of the 14th ACM International Symposium on QoS and Security for Wireless and Mobile Networks (Q2SWinet'18). 29-38.

[41] SAE. 2017. Aerospace Standard AS5506C: Architecture Analysis and Design Language (AADL) (2017). Standard AS5506C.

[42] Bran Selic. 1998. Using UML for modeling complex real-time systems. In Proceedings of the Annual Conference on Languages, Compilers, Tools and Theory of Embedded Systems (LCTES'98). Springer, 250-260.

[43] Bran Selic and Sébastien Gérard. 2013. Modeling and Analysis of Real-Time and Embedded Systems with UML and MARTE: Developing Cyber-Physical Systems. Elsevier.

[44] Aviral Shrivastava, Patricia Derler, Ya-Shian Li-Baboud, Kevin Stanton, Mohammad Khayatian, Hugo A. Andrade, Marc Weiss, John C. Eidson, and Sundeep Chandhoke. 2016. Time in cyber-physical systems. In Proceedings of the 11th IEEE/ACM/IFIP International Conference on HW/SW Codesign and System Synthesis (CODES'16). 4:14:10.

[45] CCITT Specification. 1999. Specification and description language (SDL). ITU-T Recommendation Z.100. https://www. itu.int/ITU-T/studygroups/com10/languages/Z.100_1199.pdf.

[46] Simone Spitz et al. 1997. SDL*-an annotated specification language for engineering multimedia communication systems. In Proceedings of the 6th Open Workshop on High Speed Networks. Citeseer. 
[47] Lothar Thiele, Karsten Strehl, Dirk Ziegenbein, Rolf Ernst, and Jürgen Teich. 1999. FunState - an internal design representation for codesign. In Proceedings of the IEEE/ACM Int. Conference on Computer-Aided Design. 558-565.

[48] Thomas Wiltschko. 2004. Sichere Information durch infrastrukturgestützte Fahrerassistenzsysteme zur Steigerung der Verkehrssicherheit an Straßenknotenpunkten. Ph.D. dissertation. Universität Stuttgart.

Received April 2019; revised October 2019; accepted February 2020 